ARTICLE

Received 7 Aug 2015 | Accepted 28 Oct 2015 | Published 4 Dec 2015

DOI: $10.1038 /$ ncomms10027

OPEN

\title{
Concurrent BMP7 and FGF9 signalling governs AP-1 function to promote self-renewal of nephron progenitor cells
}

Sree Deepthi Muthukrishnan ${ }^{1,2}$, Xuehui Yang $^{1}$, Robert Friesel ${ }^{1} \&$ Leif Oxburgh ${ }^{1}$

Self-renewal of nephron progenitor cells (NPCs) is governed by BMP, FGF and WNT signalling. Mechanisms underlying cross-talk between these pathways at the molecular level are largely unknown. Here we delineate the pathway through which the proliferative BMP7 signal is transduced in NPCs in the mouse. BMP7 activates the MAPKs TAK1 and JNK to phosphorylate the transcription factor JUN, which in turn governs transcription of AP-1element containing G1-phase cell cycle regulators such as Myc and Ccnd1 to promote NPC proliferation. Conditional inactivation of Tak1 or Jun in cap mesenchyme causes identical phenotypes characterized by premature depletion of NPCs. While JUN is regulated by BMP7, we find that its partner FOS is regulated by FGF9. We demonstrate that BMP7 and FGF9 coordinately regulate AP-1 transcription to promote G1-S cell cycle progression and NPC proliferation. Our findings identify a molecular mechanism explaining the important cooperation between two major NPC self-renewal pathways.

\footnotetext{
${ }^{1}$ Center for Molecular Medicine, Maine Medical Center Research Institute, 81 Research Drive, Scarborough, Maine 04074, USA. ${ }^{2}$ Graduate School of Biomedical Sciences and Engineering, University of Maine, Orono, Maine 04469, USA. Correspondence and requests for materials should be addressed to L.O. (email: Oxburl@mmc.org).
} 
terative nephron induction is based on a program of reciprocal interactions between the ureteric bud and the surrounding nephron progenitor cells (NPCs) of the cap mesenchyme. The cap mesenchyme is divided into compartments expressing distinct transcription factors. The highest order compartment expresses $\mathrm{Cbp} / \mathrm{p} 300$-interacting transactivator 1 (CITED1) and Six homeobox 2 (SIX2). This compartment transitions to the CITED1 - /SIX2 + compartment that subsequently differentiates into the pre-tubular aggregate, the precursor of the epithelial renal vesicle ${ }^{1,2}$. A fine balance between NPC self-renewal and differentiation is critical in determining nephron number in the adult kidney. Three major growth factor pathways are essential for NPC maintenance and self-renewal: Wingless-type MMTV integration site family member 9B (WNT9B) / $\beta$-catenin, fibroblast growth factors (FGF) $9 / 20$, and bone morphogenetic protein-7 (BMP7)/MAPK (refs 3-7). Previous reports suggest important signalling interactions in NPCs. For example, BMP and FGF synergistically promote progenitor cell maintenance in organ culture ${ }^{8}$. Understanding the mechanistic bases for these interactions is important to advance our understanding of renal organogenesis and for attempts at de novo nephrogenesis from stem cells.

To define the circuitry connecting signalling pathways and thus build an integrated model for regulation of NPC self-renewal, we first need to map signal transduction mechanisms used by each growth factor. In this study, we define the MAPK signalling cascade that transduces the proliferative response to BMP7 using complementary primary cell culture and conditional gene inactivation approaches. We show that the BMP7 signal is transduced through TAK1 and JNK to activate the transcription factor JUN in NPCs. JUN is required for proliferation of these cells and directly governs G1-phase cell cycle regulatory genes including $M y c$ and Ccnd1. JUN is a component of the dimeric AP-1 transcription factor that also includes FOS (ref. 9). AP-1 components are differentially regulated by growth factor stimuli, and changes in dimer composition strongly influence AP-1 function ${ }^{10}$. Using both genetic and primary cell models we show that BMP7 and FGF9 have distinct effects on JUN and FOS. While FGF9 controls FOS activation, BMP7 activates JUN, which is the DNA-binding partner in the JUN-FOS heterodimer. JUN-FOS heterodimers are strong transactivators with enhanced DNA-binding ability compared with JUN homodimers ${ }^{10}$. Compared with either BMP7 or FGF9 treatment, combined BMP7 and FGF9 stimulation of NPCs amplifies activation of an AP-1 transcriptional reporter, increases transcription of the G1-S cell cycle regulator Ccnd1, and promotes G1-S transition and proliferation of cells. On the basis of these findings we propose that BMP7 and FGF9 cooperatively control AP-1 function to promote NPC proliferation, providing an explanation for the important synergy between these growth factors in NPC maintenance.

\section{Results}

BMP7 promotes NPC proliferation through TAK1 and JNK. Previous work from our laboratory indicated that BMP7 promotes proliferation of cells in the nephrogenic zone through MAPK signalling7. To determine the kinetics of pathway activation specifically in NPCs, we measured the phosphorylation states of each of the MAPK components TAK1, JNK and JUN in response to BMP7 in NPCs purified by immunomagnetic separation (Fig. 1a) ${ }^{2,11}$. Following BMP7 treatment, we detected a step-wise sequence of phosphorylation events with peak activation of pTAK1 at $10 \mathrm{~min}$, pJNK at $15 \mathrm{~min}$ and pJUN at $20 \mathrm{~min}$ (Fig. 1b,c and Supplementary Fig. 1a). Activated JUN binds to AP-1 elements in target genes including itself and $M y c$ (refs 12,13). In NPCs, Jun and $M y c$ were upregulated $2 \mathrm{~h}$ after BMP7 stimulation and pre-treatment with TAK1 and JNK inhibitors significantly reduced this response, indicating that they are early transcriptional targets of the pathway in these cells (Fig. 1d). Tak1- and Jun-deficient NPCs showed a significant reduction in BMP7 stimulation of Jun and $M y c$ transcription, corroborating the finding that BMP7 controls transcription of Jun and $M y c$ through TAK1-JNK signalling (Fig. 1e,f). Myc is required for renewal of NPCs in vivo, and our findings outline one signalling mechanism for the control of $M y c$ expression in these cells ${ }^{14}$.

To evaluate the role of the BMP7-TAK1-JNK-JUN pathway in cellular proliferation, we assessed the growth curves of BMP7stimulated NPCs treated with inhibitors of TAK1 or JNK. As expected, BMP7-stimulated proliferation was reversed by TAK1 or JNK inhibition (Fig. 1g). To confirm that NPCs retained their phenotype in the experimental conditions, we measured expression of CITED1, SIX2 and LEF1 as well as evaluating a panel of markers for cap mesenchyme and cortical interstitium (Supplementary Fig. 1b,c). To confirm that BMP7-stimulated proliferation depends on kinase activity of pathway components, wild type (WT) and kinase-dead versions of TAK1 and JUN were expressed in NPCs, which were stimulated with BMP7. Transfection efficiency was analysed by expressing a GFP construct, and by measuring the expression of Tak1 and Jun transcripts in transfected NPCs (Supplementary Fig. 1d,e). Wild type TAK1 and JUN expression augmented the BMP7-induced proliferative response, whereas kinase-dead variants reduced it, confirming that phosphorylation of pathway components is essential for proliferation of NPCs (Fig. 1h and Supplementary Fig. 1f). On the basis of our primary cell analysis we conclude that BMP7 promotes NPC proliferation through activation of the TAK1-JNK-JUN signalling cascade.

Bmp7 and Tak1 interact to control NPC renewal. To confirm the BMP7-TAK1 relationship in vivo, we used the $B m p 7^{+/ \text {cre }}$ strain to inactivate a single copy of Tak1. Bmp $7^{+/ \text {cre }}$ is an inactivating mutation and heterozygous animals express only one copy of the gene ${ }^{15}$. We reasoned that limiting the availability of TAK1 would exacerbate the effect of reduced BMP7 ligand availability if these molecules operate in the same pathway. Although the body weight of $B m p 7^{+/ c r e}$ embryos appeared slightly lower than WT, no difference could be detected between $B m p 7^{+/ c r e}$ and $B m p 7^{+/ c r e} ; T a k 1^{+/ c}$ mice (Supplementary Fig. 2a). Morphometric analyses at E14.5 and P0 revealed significant reductions in size $\left({ }^{\star} P<0.05\right)$ and weight $\left({ }^{*} P<0.001\right.$, Student's $t$-test, $\left.n=6\right)$ of $B m p 7^{+/ \text {cre }} ;$ Tak $1^{+/ c}$ kidneys compared with $B m p 7^{+/ / c r e}$, supporting the notion that BMP7 indeed does signal through TAK1 in vivo (Fig. 2a,b and Supplementary Fig. 2b,c). To verify loss of Tak1 and Jun in mutant kidneys, we measured expression of Tak1, Jun, and their downstream target $M y c$ in isolated NPCs from P0 WT, Tak1 ${ }^{+/ c}$, $B m p 7^{+/ c r e}$ and Bmp $7^{+/ / \text {re }} ;$ Tak1 $1^{+/ c}$ mice. Tak1, Jun and Myc were reduced by $\sim 60 \%$ in $B m p 7^{+/ / c r e} ;$ Tak $1^{+/ c}$ compared with WT (Fig. 2c). Activated pJUN levels were decreased in the cap mesenchyme, but not in the collecting duct tips of $B m p 7^{+/ / c r e}$; Tak $1^{+/ c}$ kidneys compared with $B m p 7^{+/ / c r e}$ and WT, confirming that compound heterozygosity for $B m p 7$ and Tak1 results in reduced activation of JNK-JUN signalling specifically in NPCs (Supplementary Fig. 2d,e).

Cell death in the nephrogenic zone and premature loss of NPCs are hallmarks of the Bmp7 null mutants ${ }^{16-18}$. We therefore measured proliferation and cell death in NPCs of single and compound mutants. Using SIX2 with the proliferation marker Ki67, we observed a marked reduction in Ki67 + /SIX2 + cells at 


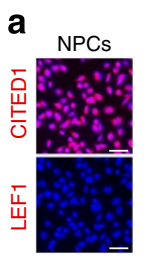

b

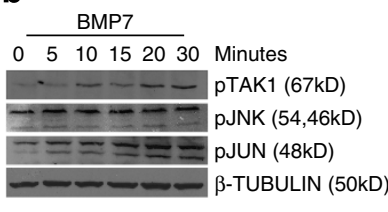

e

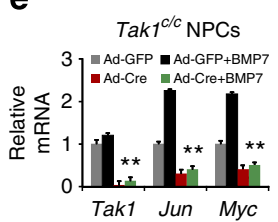

f

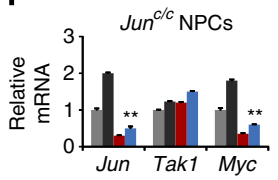

g

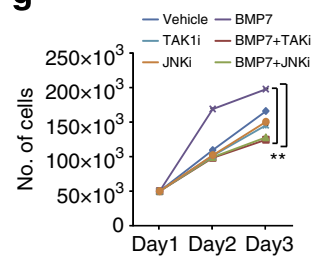

C

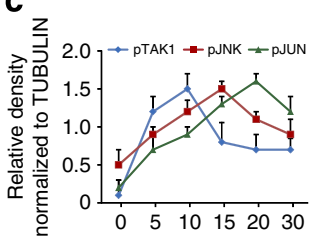

d

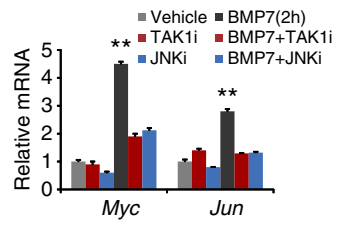

h

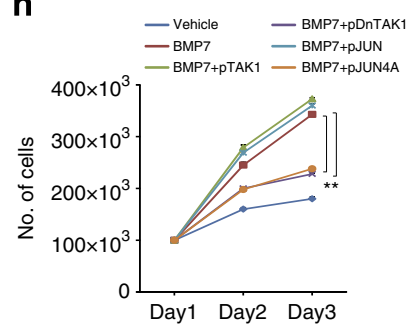

Figure 1 | BMP7 activates TAK1-JNK-JUN signalling in NPCs. (a) CITED1 and LEF1 immunostaining of freshly purified E17.5 NPCs. Scale bars, $50 \mu$ M. (b) Immunoblot shows time course of activation of pTAK1, pJNK and pJUN in NPCs stimulated with BMP7 (Supplementary Fig. 1a). (c) Average protein densities measured at each time point from two independent experiments $(n=2)$. Error bars represent s.d. (d) RT-qPCR of Myc and Jun on NPCs treated with vehicle, BMP7, TAK1 and JNK inhibitors for $2 \mathrm{~h}$. Error bars represent s.d. ${ }^{\star \star} P<0.005$, Student's $t$-test. Three biological replicates analysed per condition, $n=3$. (e,f) RT-qPCR of Tak1, Jun and Myc in Tak1- and Jun-inactivated NPCs stimulated with BMP7 for 2 h. Error bars represent s.d. ${ }^{\star \star} P<0.005$, Student's t-test. Two biological replicates analysed per condition, $n=2$. (g) Growth curve of NPCs treated with vehicle, BMP7, TAK1 and JNK inhibitors. Average numbers are derived from five biological replicates per condition, $n=5$. Error bars represent s.d. ${ }^{\star \star} P<0.005$, Student's $t$-test. (h) Growth curve of vehicle or BMP7-treated NPCs transfected with WT or kinase-dead constructs of TAK1 and JUN. Average numbers are derived from three biological replicates per condition, $n=3$. Error bars represent s.d. ${ }^{\star \star} P<0.009$ and ${ }^{\star \star} P<0.005$, Student's $t$-test.

E14.5 (15\%) and P0 (10\%), and a concomitant decrease in the number of SIX $2+$ cells per kidney in the $B m p 7^{+/ c r e} ;$ Tak $1^{+/ c}$ kidneys relative to the $B m p 7^{+/ c r e}$ mutant (Fig. $2 \mathrm{~d}-\mathrm{g}$ and Supplementary Fig. 2f-i). Apoptosis analysis showed no evidence of increased cell death in E14.5 Bmp $7^{+/ \mathrm{cre}} ; \mathrm{Tak} 1^{+/ \mathrm{c}}$ or $B m p 7^{+/ c r e}$ kidneys, suggesting that Tak1 is involved only in the proliferative response of NPCs to BMP7 (Supplementary Fig. 2j). Growth and branching of the collecting duct is controlled by factors secreted by NPCs, and to determine if branching was secondarily affected in $B m p 7^{+/ c r e} ; T a k 1^{+/ c}$ kidneys, we quantified the number of collecting duct tips. Bmp $7^{+/ c r e}$ kidneys show reduced branching relative to the WT, which strongly suggests an effect of diminished NPC numbers in this mutant considering that the reduction of $B m p 7$ caused by heterozygosity is predicted to promote ureteric bud outgrowth and branching ${ }^{19,20}$. Compared with $B m p 7^{+/ c r e}, B m p 7^{+/ c r e} ;$ Tak $1^{+/ c}$ kidneys showed a further reduction of collecting duct branching proportional to the reduction in NPC number (Fig. $2 \mathrm{~h}$ and Supplementary Fig. 2k). Overall, our genetic interaction study supports control of NPC renewal by BMP7 signalling through TAK1 in the developing kidney.

Deletion of Tak1 and Jun in NPCs reduces their renewal. To stringently determine the requirement for components of the BMP7-TAK1-JNK-JUN pathway in NPCs in vivo, we inactivated Tak1 and Jun using Six2-cre. Both Tak1 ${ }^{N P C}\left(\right.$ Six2-cre;Tak1 $\left.{ }^{c / c}\right)$ and $J_{u n}{ }^{N P C}\left(\right.$ Six2-cre; Jun $\left.{ }^{c / c}\right)$ P0 kidneys showed significant reduction in kidney weight (50\%) and size (30-40\%) compared with Tak1 $^{\text {het }}$ (Six2-cre;Tak1 ${ }^{+/ c}$ ) and Jun ${ }^{\text {het }}$ (Six2-cre;Jun ${ }^{+/ c}$ ) kidneys ${ }^{* \star} P<0.005$, Student's $t$-test), confirming that these genes are essential in the Six2 lineage, which is limited to NPCs and their derivatives (Fig. 3a-c and Supplementary Fig. 3a) ${ }^{21}$. Body weights of these different strains did not show significant differences
$(P>0.05$, Student's $t$-test; Supplementary Fig. $3 \mathrm{~b})$. To verify loss of Tak1 and Jun, we measured expression of Tak1, Jun and their target $M y c$ in NPCs isolated at E17.5 (Tak1 ${ }^{N P C}$ and Tak1 het $)$ or E14.5 (Jun $^{N P C}$ and Jun ${ }^{\text {het }}$ ). Tak1 and Jun were reduced by $80 \%$ and $M y c$ by $\sim 50-60 \%$ in Tak1 ${ }^{\text {NPC }}$ and Jun ${ }^{N P C}$ NPCs, respectively (Fig. 3d,e). As expected, Tak1 was unchanged in $J u n^{N P C}$ NPCs (Fig. 3e). pJUN and MYC were reduced in mutant kidneys, confirming that inactivation of Tak1 and Jun results in reduced activation of JNK-JUN signalling and downstream targets in NPCs (Fig. 3f and Supplementary Fig. 3c).

Morphologically, Tak $1^{N P C}$ and Jun ${ }^{N P C}$ kidneys revealed several atypically organized cap mesenchymes carrying few NPCs (Fig. 3g and Supplementary Fig. 3d,e). SIX2 + cells were reduced by $\sim 50 \%$ in mutant kidneys, suggesting that Tak1 and Jun inactivation results in premature loss of NPCs (Fig. 3h and Supplementary Fig. 3e). To understand if this was due to reduced proliferation, we measured coexpression of $\mathrm{Ki} 67$ or $\mathrm{pHH} 3$ and SIX2 in Tak1 ${ }^{N P C}(\mathrm{P} 0)$ and Jun ${ }^{N P C}$ (E14.5 and P0) kidneys. We observed $50 \%$ reduction of $\mathrm{Ki} 67+/ \mathrm{SIX} 2+$ cells and $\mathrm{pHH} 3+/ \mathrm{SIX} 2+$ cells in Tak1 ${ }^{N P C}(\mathrm{P} 0)$ and $J u n^{N P C}(\mathrm{E} 14.5, \mathrm{P} 0)$ kidneys (Fig. 3i,j and Supplementary Fig. 3e). TUNEL and caspase3 staining showed no evidence of cell death in the Tak $1^{N P C}$ kidneys, suggesting that loss of NPCs was strictly due to reduced proliferation (Supplementary Fig. 3f).

To rule out the possibility that Tak1 and Jun mutant NPCs may take on a cortical interstitial fate, we analysed Tak1 ${ }^{N P C}$ (E17.5) and $J u n^{N P C}$ (E14.5) NPCs for markers of cap mesenchyme (Cited1, Six2, Dpf3 and Meox1) and cortical interstitium (Foxd1 and Sfrp1). Cap mesenchyme markers either remained unchanged or showed a slight increase in Tak1 ${ }^{N P C}$ (E17.5) and Jun ${ }^{N P C}$ (E14.5). However, neither Foxd1 nor Sfrp1 were elevated in Tak1 $1^{N P C}$ or Jun ${ }^{N P C}$ indicating that the cellular identity of NPCs is unaltered (Fig. 3d,e and Supplementary Fig. 3g). To confirm that Tak1 and Jun mutant NPCs retain their 
a

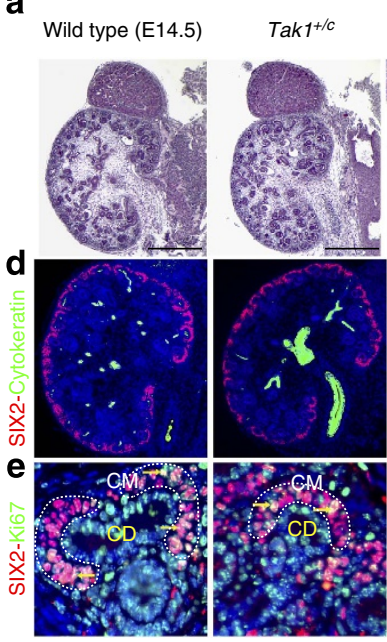

$B m p 7^{+/ c r e} \quad B m p 7^{+/ c r e .} \cdot T a k 1^{+/ c}$

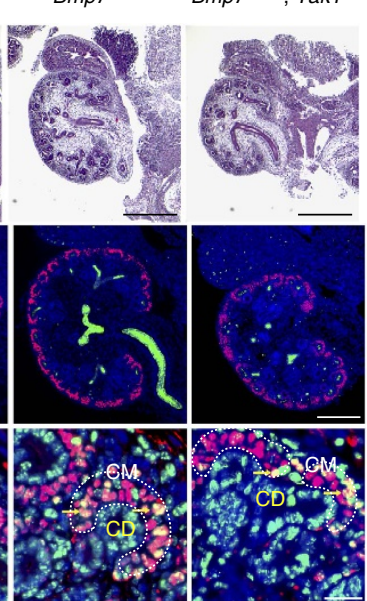

b

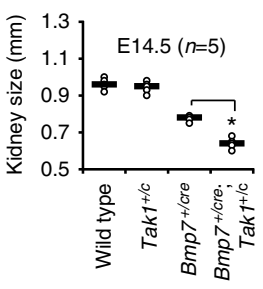

C

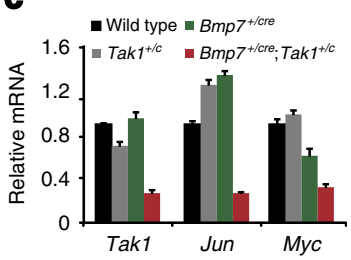

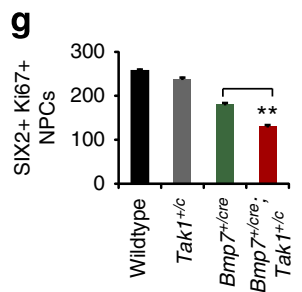

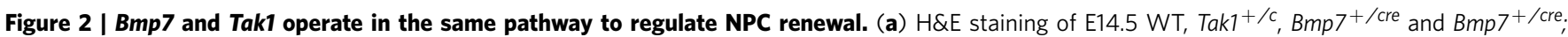
Tak $1^{+/ c}$ kidneys. Scale bars, $500 \mu \mathrm{M}$. (b) Kidney sizes. Number of mice analysed per genotype is noted in the graph. ${ }^{\star} P<0.01$, Student's $t$-test.

(c) RT-qPCR of NPCs from PO kidneys for Tak1, Jun and Myc in mutants and WT control. Error bars represent s.d. Two biological replicates analysed per genotype, $n=2$. (d) SIX2 (red, cap mesenchyme) and cytokeratin8 (green, collecting duct) immunostaining of whole kidney sections. Scale bars, $500 \mu \mathrm{M}$. (e) Co-immunostaining of SIX2 and the proliferation marker Ki67. Scale bars, $50 \mu \mathrm{M}$. (f-h) Quantitation of SIX2 +, SIX2+/Ki67 +, and collecting duct tips from 5 to 10 serial sections per kidney per genotype. Arrows point to Ki67+/SIX2 + cells in the cap mesenchyme, which is highlighted by white dashed lines. Error bars represent s.d. ${ }^{\star} P<0.005$ and ${ }^{\star} P<0.05$, Student's $t$-test. $C D$, collecting duct; $C M$, cap mesenchyme.

cellular identity in vivo, we performed lineage tracing analysis by crossing the Six2 $2^{+/ \text {cre }} ; \mathrm{Tak}^{+/ c}$ and Six2 ${ }^{+/ \text {cre }} ; \mathrm{Jun}^{+/ c}$ mice with the R26RLacZ reporter. $\beta$-galactosidase and SIX2 immunostaining revealed that tagged cells were confined to SIX2 + cap mesenchyme and its derivatives in both Tak1 $1^{\text {NPC }}$ and Jun ${ }^{N P C}$ kidneys (Fig. 3k and Supplementary Fig. 3h). Thus, inactivation of Tak1 and Jun in NPCs partially phenocopies the Bmp7 null phenotype, suggesting that they operate in the same pathway to regulate NPC self-renewal.

The few $\mathrm{Ki} 67+/$ SIX2 + NPCs we observed in Tak1 ${ }^{\text {NPC }}$ kidneys localized predominantly to the distal cap mesenchyme under the collecting duct tips, in which CITED1 expression is normally lost (see insets in Fig. 3i). To understand if the Tak $1^{N P C}$ phenotype results from gene inactivation specifically in the CITED1 + compartment, we used the Cited1-creER ${ }^{T 2}$ strain. Cited1-creER ${ }^{T 2} ;$ Tak $^{c / c}\left(\right.$ Takl $\left.1^{C-N P C}\right)$ and Tak1 $1^{c / c}$ (Tak1 ${ }^{C-W T}$, littermate control) mice were tamoxifen injected at either E11.5 or E14.5 and collected after $72 \mathrm{~h}$. Tak1 ${ }^{C-N P C}$ kidneys were significantly smaller than Takl ${ }^{C-W T}$ at both time points $\left({ }^{\star \star} P<0.005\right.$, Student's $t$-test), and their cap mesenchymes were depleted (Fig. 4a and Supplementary Fig. 4a,b). Tak1 transcript was reduced by $80 \%$ and both Jun and $M y c$ were reduced in mutant NPCs (Fig. 4b). Immunoblotting confirmed the reduction of TAK1 in mutant NPCs (Fig. 4c and Supplementary Fig. 4c). pJUN and MYC were also reduced, indicating that Tak1 inactivation results in reduced JNK-JUN signalling in NPCs (Fig. $4 \mathrm{~d}$ and Supplementary Fig. $4 \mathrm{~d}, \mathrm{e}$ ). To understand if Tak1 is required to maintain NPCs in the CITED1+/SIX2 + state, we analysed cap mesenchyme markers in $\mathrm{Tak1}^{\mathrm{C}-\mathrm{NPC}}$ NPCs. Tak1-inactivated NPCs maintained expression of Cited 1 and Six2 at levels similar to WT, indicating that they retain the appropriate cellular identity. However, the number of CITED1 + NPCs was reduced by $25-30 \%$ at both E14.5 and E17.5 (Fig. 4e-g and Supplementary Fig. 4f). Co-immunostaining for CITED1, SIX2 and $\mathrm{pHH} 3$ confirmed decreased $\mathrm{pHH} 3$ staining in CITED1 +1 SIX2 + cells of Tak1 ${ }^{C-N P C}$ kidneys relative to Tak $1^{C-W T}$ but no difference in the CITED1 - /SIX2 + compartment at either E14.5 or E17.5, validating our findings from Tak1 ${ }^{N P C}$ mutants (Fig. $4 \mathrm{~h}, \mathrm{i}$ and Supplementary Fig. 4g). Collectively, our genetic analysis suggests that the BMP7-TAK1-JNK-JUN pathway is required for proliferation of the early CITED1 + /SIX2 + compartment in vivo.

BMP7 promotes G1 to $S$ cell cycle progression in NPCs. Having defined the requirements for components of the BMP7-TAK1JNK-JUN signalling cascade in NPCs, we wanted to understand how this pathway interfaces with cellular proliferation control mechanisms. We have shown that the BMP7-TAK1-JNK-JUN pathway activates Jun and $M y c$ transcription in NPCs (Figs 3 and 4). JUN and MYC are key transcriptional regulators of the cell cycle that modulate expression of genes involved in the G1-phase ${ }^{22,23}$. To test how the BMP7-TAK1-JNK-JUN pathway controls NPC proliferation, we investigated the effects of inhibiting pathway components on G1-S transition in BMP7stimulated NPCs. Immunostaining for specific markers of G1 (CCNE1) and S (PCNA) was performed to calculate the percentages of G1 and S-phase cells in each experimental condition. After $24 \mathrm{~h}$ stimulation, BMP7 robustly promoted G1 to $S$ transition in both E14.5 and E17.5 NPCs (Fig. 5a,b). TAK1 or JNK inhibition reversed this effect, confirming that BMP7 
a
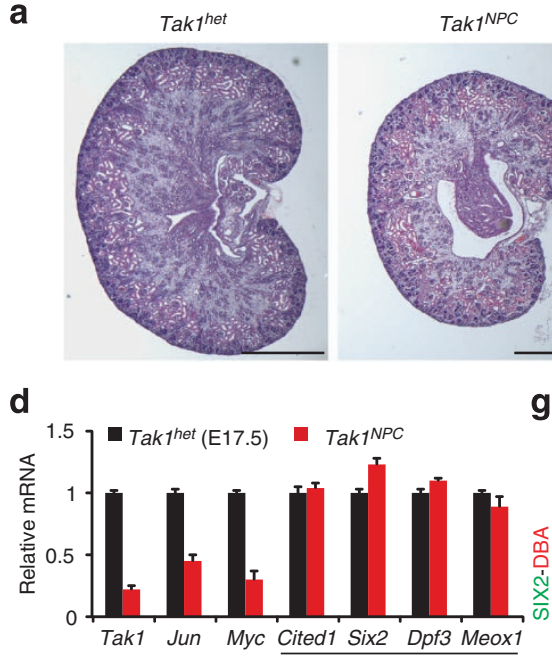

e

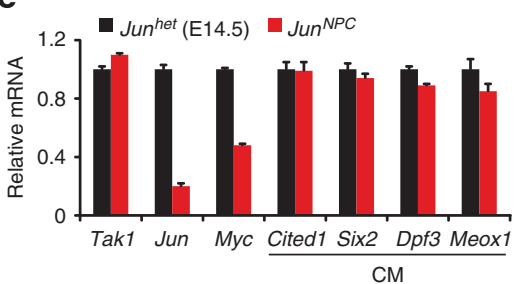

f

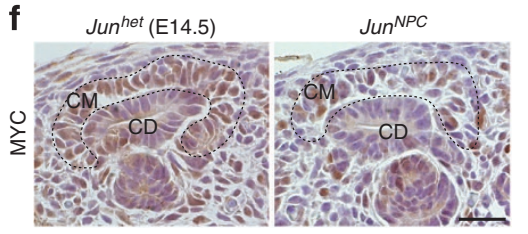

b
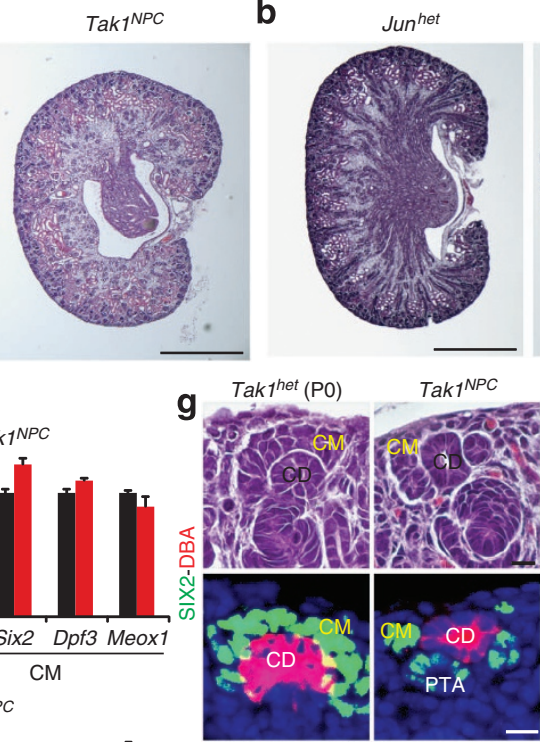

Tak1 $^{\text {het }}(\mathrm{P} 0)$

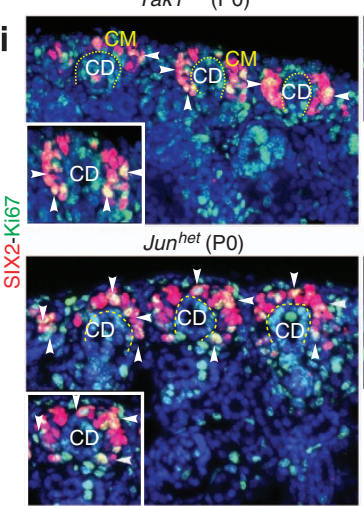

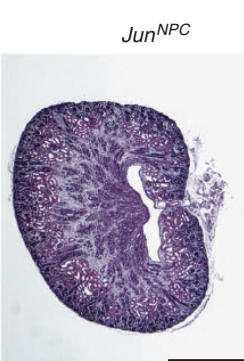

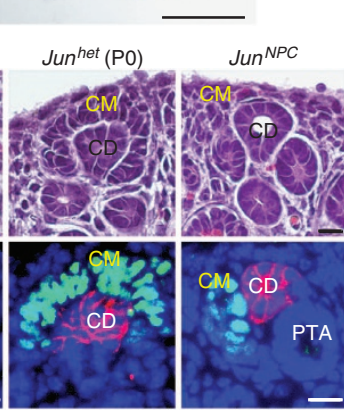

$\operatorname{Tak}^{N P C}$

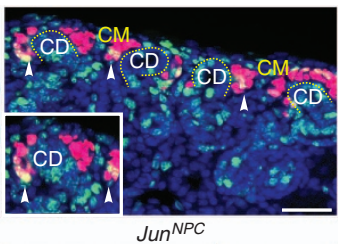

$J^{N}{ }^{N P C}$

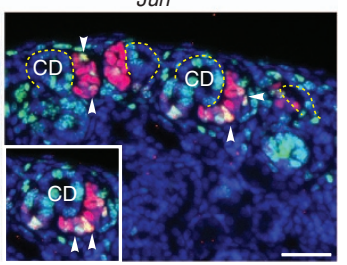

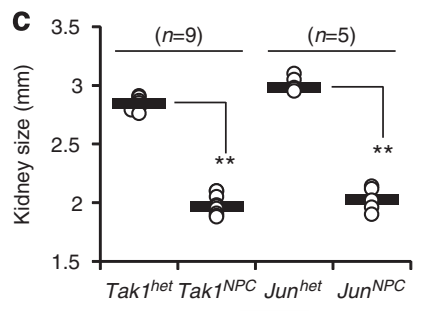
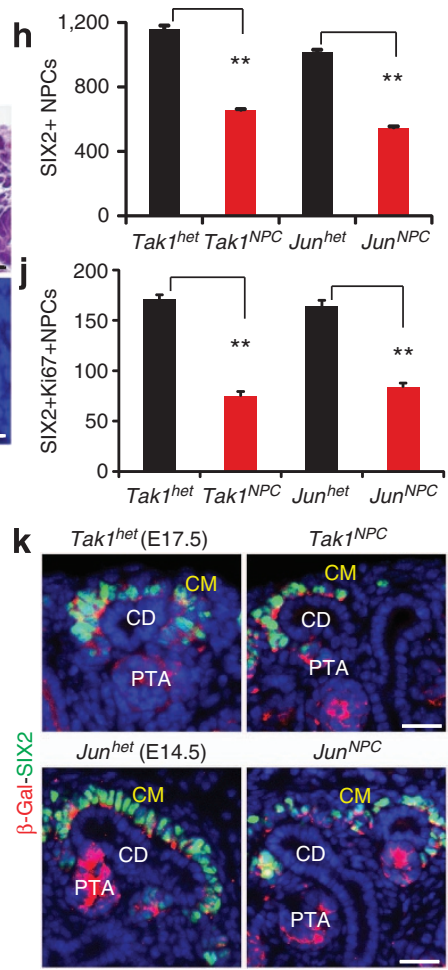

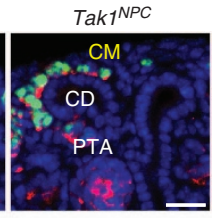

Jun NPC

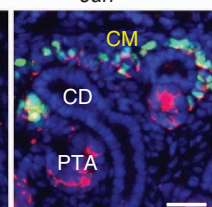

Figure 3 | Tak1 and Jun are required for self-renewal of NPCs throughout nephrogenesis. (a,b) H\&E staining of PO Tak $7^{\text {het }}$, Tak $7^{N P C}$, Jun ${ }^{\text {het }}$ and Jun $^{N P C}$ kidneys. Scale bars, $500 \mu \mathrm{M}$ (c) Kidney sizes. Number of mice (n) analysed per group is noted in the graph. ${ }^{\star \star} P<0.005$, Student's $t$-test. (d,e) Transcriptional analysis of Tak1, Jun, Myc and cap mesenchyme markers in NPCs isolated from E17.5 Tak ${ }^{\text {het, }}$, Tak $7^{N P C}$ and E14.5 Jun ${ }^{\text {het }}$, Jun ${ }^{\text {NPC }}$ kidneys. Error bars represent s.d. Two biological replicates analysed per genotype, $n=2$. (f) MYC immunostaining of E14.5 Jun ${ }^{\text {het }}$ and Jun NPC kidneys. Scale bars, $50 \mu \mathrm{M}$. (g) H\&E and co-immunostaining of SIX2 (green, cap mesenchyme) and DBA lectin (red, collecting duct) of PO Tak7 $7^{N P C}$ and Jun ${ }^{N P C}$ kidneys. Scale bars, $25 \mu \mathrm{M}$. (h) Average number of SIX2 + NPCs per kidney section per genotype. Error bars indicate s.d. ${ }^{\star \star} P<0.005$, Student's t-test. (i) Ki67 (green) and SIX2 (red) co-immunostaining of PO Tak $7^{\text {het }}$, Tak $7^{N P C}$, Jun het, Jun NPC kidneys. Insets show magnifications of cap mesenchymes with arrows pointing to Ki67 + cells. Scale bars, $100 \mu \mathrm{M}$. (j) Number of SIX2 + /Ki67 + cells per kidney section. Error bars represent s.d. and ${ }^{\star \star} P<0.005$, Student's $t$ -

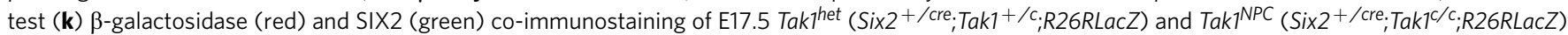
kidneys. Three mice were analysed per genotype at E14.5 and E17.5 $(n=3)$. CD, collecting duct; CM, cap mesenchyme; PTA, pre-tubular aggregate.

promotes NPC proliferation by controlling G1 to S transition through TAK1-JNK-JUN signalling (Fig. 5a,b and Supplementary Fig. 5a,b).

To understand the mechanism underlying the effect of BMP7TAK1-JNK-JUN signalling on the G1-S transition, we set out to define the repertoire of G1-phase cell cycle regulatory genes modulated by the pathway in NPCs. Several cell cycle regulators containing AP-1-binding sites are JUN targets including Ccnd1, Ccnd3, p21, p16, Jun and Myc (refs 24,25). Like JUN, MYC regulates the cell cycle by controlling G1-phase genes. Although a number of targets are shared, MYC has a unique repertoire including Ccne1, Cdc25a, p27 and Ccna2 (ref. 22). BMP7 may therefore control G1-S cell cycle regulators not only through JUN but also through MYC. Conditional gene inactivation shows that $M y c$ is required for NPC renewal at E15.5-E18.5 but not earlier in nephrogenesis, suggesting that the contribution of MYC to cell cycle control by BMP7 might be limited to later stages of nephrogenesis ${ }^{14}$. To understand if this is the case, we compared the responsiveness of MYC and JUN targets to BMP7 in NPCs at E14.5 and E17.5. The JUN targets Ccnd1, Cond3 and p21 were regulated by BMP7 in a TAK1- and JNK-dependent manner in both E14.5 and E17.5 NPCs (Fig. 5c,d). However, the MYC targets Ccne1, Cdc25a and p27 were regulated by BMP7 in a TAK1 - and JNK-dependent manner only in E17.5 NPCs (Fig. 5c,d and Supplementary Fig. 5c-f). Our analysis indicates that the BMP7-TAK1-JNK-JUN pathway regulates JUN cell cycle targets including $M y c$ throughout nephrogenesis, but that the contribution of MYC itself to control of G1 targets is limited to later stages of nephrogenesis.

To confirm these observations in vivo, we first measured target gene activation in NPCs isolated from E14.5 Jun ${ }^{N P C}$ and E17.5 Tak $1^{N P C}$ kidneys. As expected, JUN targets were misregulated in mutant NPCs at both E14.5 and E17.5, whereas MYC targets were misregulated only at E17.5 (Fig. 5e,f). Next, we immunostained Bmp7 null, Jun ${ }^{N P C}$ and Tak1 ${ }^{C-N P C}$ kidneys for the JUN-activated target CCND1 and the MYC-activated 
a
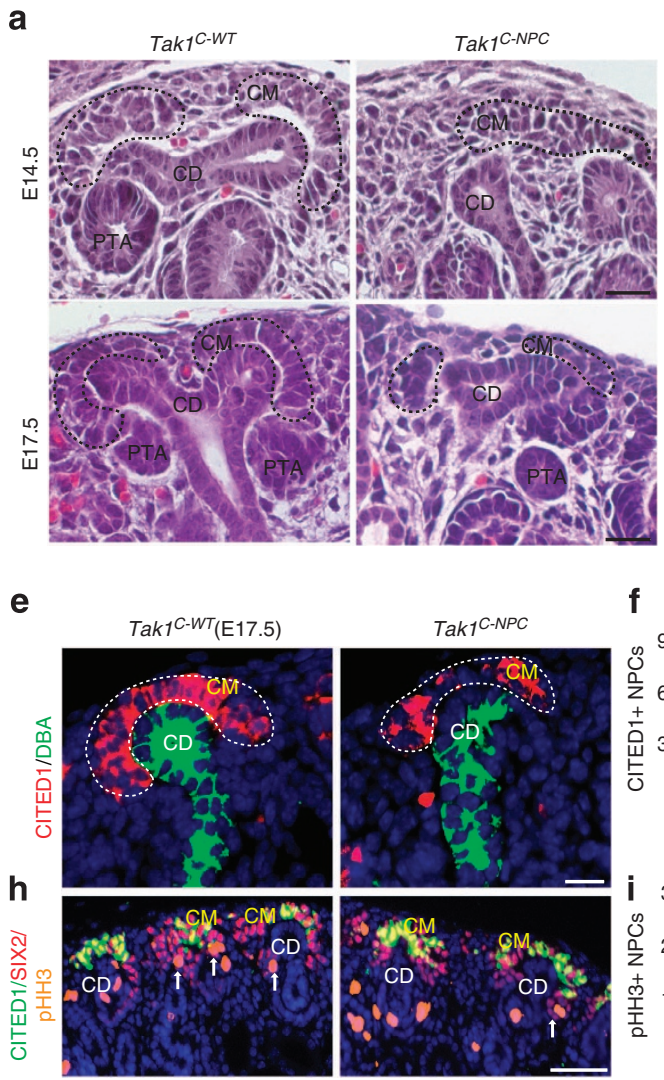

f

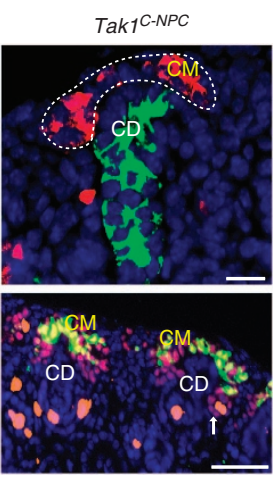

b

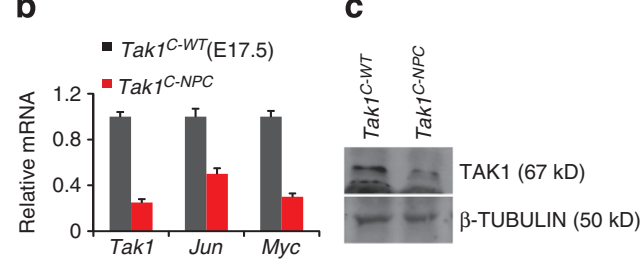

d
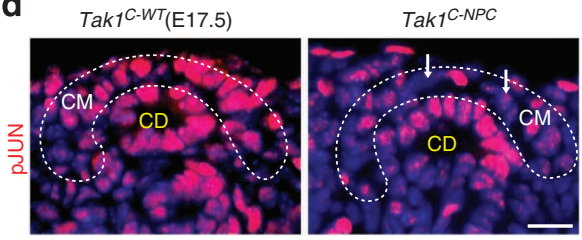
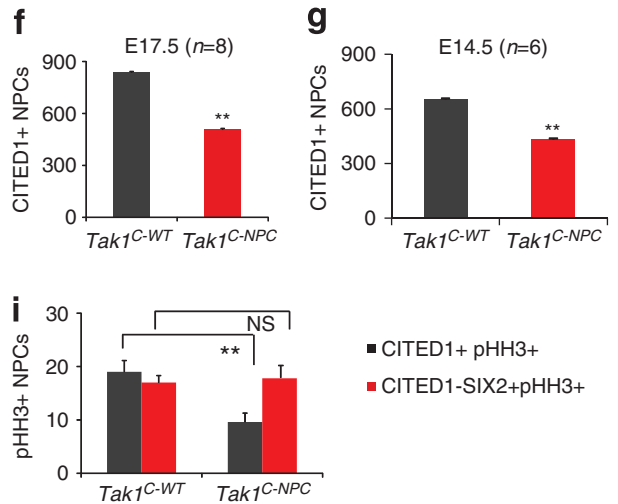

- CITED1+ pHH3+

- CITED1-SIX2+pHH3+

Figure 4 | BMP7-mediated TAK1 signalling is required for proliferation of CITED1 + NPCs. (a) H\&E staining of E14.5 and E17.5 Tak7 $7^{C-W T}$ and Tak $7{ }^{C-N P C}$ kidneys shows nephrogenic units in which cap mesenchymes are outlined with black dashed lines. Scale bars, $50 \mu \mathrm{M}$ (b) RT-qPCR of Tak1, Jun and Myc in NPCs isolated from E17.5 Tak $7^{C-W T}$ and Tak $7^{C-N P C}$ kidneys. Error bars indicate s.d. Two biological replicates analysed per genotype $(n=2)$. (c) Immunoblot of TAK1 and $\beta$-Tubulin in NPCs isolated from E17.5 Tak $7^{C-W T}$ and Tak ${ }^{C}$-NPC kidneys (Supplementary Fig. 4c). (d) pJUN immunostaining of E17.5 Tak $7^{C-W T}$ and Tak ${ }^{C-N P C}$ kidneys. Scale bars, $50 \mu \mathrm{M}$. (e-g) CITED1 (red) and DBA lectin (green) staining of E17.5 Tak $7^{\mathrm{C}-W T}$ and Tak ${ }^{\mathrm{C}-\mathrm{NPC}}$ kidneys. Quantitation of numbers of CITED1 + cells per E14.5 and E17.5 kidney section per genotype. Number of mice $(n)$ per genotype is noted in the graph. Error bars represent s.d. ${ }^{\star \star} P<0.005$, Student's $t$-test. (h,i) CITED1 (green), SIX2 (red) and pHH3 (orange/proliferation marker) staining in E17.5 Tak $7^{\mathrm{C}-W T}$ and Tak1 ${ }^{\mathrm{C}-N P C}$. Scale bars, $150 \mu \mathrm{M}$. Quantitation of numbers of pHH3 +/CITED1 + /SIX2 + versus pHH3 +/CITED1 - /SIX2 + cells per kidney section per genotype. Error bars indicate s.d. ${ }^{\star \star} P<0.005$ and $P=0.08$ (NS, not significant), Student's $t$-test. Cap mesenchymes highlighted with black or white dotted lines. $C D$, collecting duct; CM, cap mesenchyme; PTA, pre-tubular aggregate.

target CCNE1 at E14.5 and E17.5. CCND1 has been used as a marker of the distal tubule; therefore we first verified its expression in cap mesenchyme using two different antibodies ${ }^{26}$. CCND1 was expressed in a salt-and-pepper distribution in WT cap mesenchyme, as expected considering that its expression is limited to the G1-phase of the cell cycle (Fig. $5 \mathrm{~g}$ and Supplementary Fig. 5g). CCND1 expression was reduced in the cap mesenchymes of all mutants, suggesting that the BMP7TAK1-JNK-JUN pathway indeed controls CCND1 in vivo and regulates JUN targets both early and late in nephrogenesis (Fig. $5 \mathrm{~g}$ and Supplementary Fig. 5i). To understand if this could represent a general reduction in expression of G1 cell cycle genes in NPCs of mutant kidneys, we also measured expression of $C c n d 3$, which is expressed in a temporally overlapping manner with Ccnd1. Although RNA expression was reduced by $20 \%$, protein expression was not significantly altered in mutants, supporting the notion that CCND1 is specifically misregulated in BMP7TAK1-JNK-JUN pathway mutants in vivo (Supplementary Fig. 6h). Expression of the MYC-activated target CCNE1 was reduced in the E17.5 mutant kidneys but not in the E14.5 mutants, confirming our previous observation that MYC targets are regulated by the BMP7-TAK1-JNK-JUN pathway preferentially at later stages of nephrogenesis (Fig. $5 \mathrm{~h}$ and Supplementary Fig. 6j). From these analyses, we conclude that the
BMP7-TAK1-JNK-JUN pathway controls cellular proliferation of NPCs by regulating different G1-phase cell cycle regulators in early and later phases of nephrogenesis (Fig. 5i).

BMP7 and FGF9 cooperatively control AP-1 transcription. FGF9 has been reported to synergize with BMP7 to promote maintenance of isolated metanephric mesenchyme in vitro, however the molecular mechanism underlying this cross-talk remains unknown ${ }^{5,8}$. Metanephric mesenchyme consists of a mixture of cell types, and we first analysed proliferation in BMP7and FGF9-treated purified E17.5 NPCs to understand if the pathways intersect in this cell type. Using $5^{\prime}$-ethynyl-2'deoxyuridine (EdU) to label the S-phase and pHH3 to mark cells undergoing mitosis $(\mathrm{M})$, we measured the overall proliferation of NPCs stimulated with BMP7, FGF9 or BMP7 + FGF9. BMP7 or FGF9 stimulation showed a significant increase in EdU + and $\mathrm{pHH} 3+$ nuclei compared with vehicle, and this effect was further augmented in BMP7 + FGF9 stimulated cultures (Fig. 6a). Immunostaining and transcriptional analysis of cap mesenchyme markers showed that BMP7 + FGF9-treated cultures remained in the CITED1 + state following treatments (Supplementary Fig. 6a,b). Quantitation of number of $\mathrm{EdU}+$ and $\mathrm{pHH} 3+$ nuclei revealed a significant increase in $S$ and $M$-phase cells in BMP7 + FGF9-treated cultures relative to either BMP7 or FGF9 
a

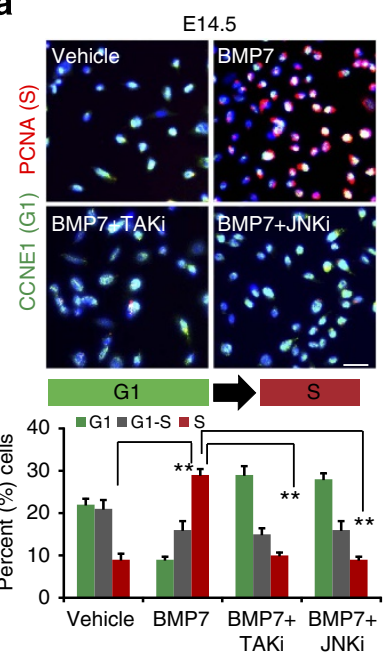

e

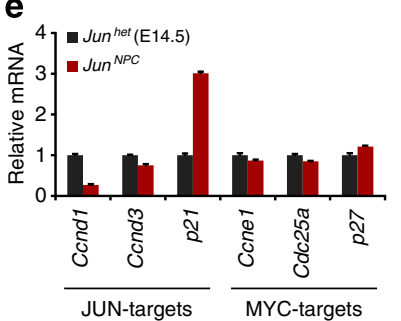

b

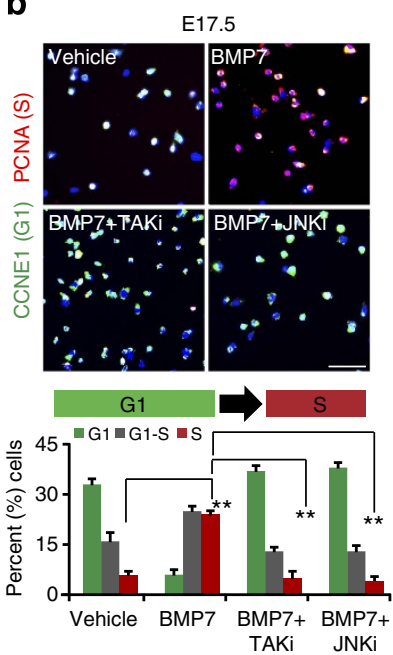

f

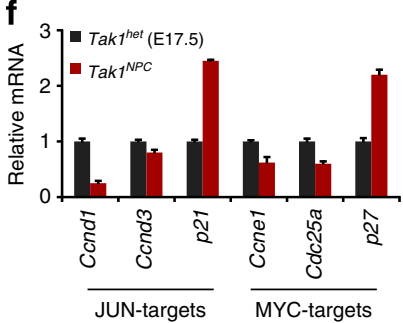

C
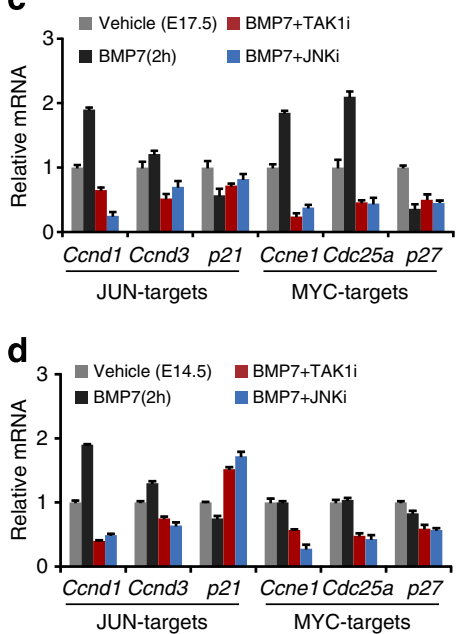

i

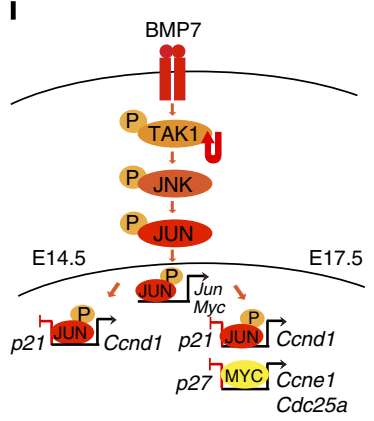

g
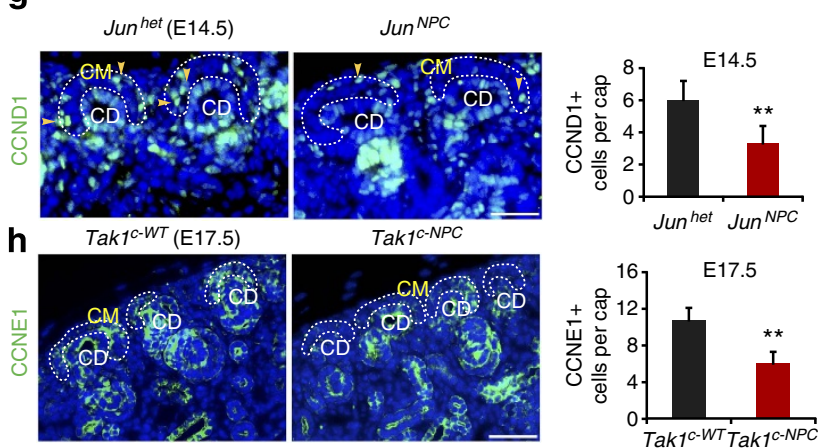

Figure 5 | BMP7 promotes G1 to S cell cycle progression in NPCs. (a,b) CCNE1 (G1-phase) and PCNA (S-phase) immunostaining of E14.5 and E17.5 NPCs treated for $24 \mathrm{~h}$ with BMP7, TAK1 and JNK inhibitors. Graphs show the percentage of G1, G1-S and S-phase cells per condition. Three biological replicates analysed per condition $(n=3)$. Error bars represent s.d. ${ }^{\star \star} P<0.005$ and ${ }^{\star \star} P<0.001$, Student's $t$-test. (c,d) RT-qPCR of JUN and MYC targets in E14.5 and E17.5 NPCs treated with vehicle, BMP7, TAK1 and JNK inhibitors for $2 \mathrm{~h}$. Error bars indicate s.d. Three biological replicates analysed per condition ( $n=3$ ). $(\mathbf{e}, \mathbf{f})$ RT-qPCR of JUN and MYC targets on NPCs isolated from E14.5 Jun ${ }^{\text {het }}$, Jun ${ }^{\text {NPC }}$ and E17.5 Tak7 $7^{\text {het }}$, Tak $7^{\text {NPC }}$ kidneys. Error bars represent s.d. Two biological replicates analysed per genotype $(n=2)$. (g,h) CCND1 and CCNE1 immunostaining in E14.5 Jun ${ }^{\text {het }}$, Jun ${ }^{N P C}$ and E17.5 Tak7 ${ }^{C-W T}$, Tak7 $7^{C-N P C}$ kidneys. Scale bars, 100 and $150 \mu \mathrm{M}$. Quantitation of CCND1 + and CCNE1 + cells per cap mesenchyme (yellow arrows) was calculated by scoring at least 30 random cap mesenchymes per kidney section per genotype for a total of 5 sections from each experimental group. Error bars indicate mean (s.d.). ${ }^{\star \star} P<0.005$ and ${ }^{\star \star} P<0.001$, Student's $t$-test. (i) Model for BMP7-TAK1-JNK-JUN pathway regulation of NPC self-renewal in early (E14.5) and later (E17.5) phases of nephrogenesis. $C D$, collecting duct; $C M$, cap mesenchyme.

stimulation, suggesting that these growth factors indeed collaboratively promote NPC proliferation (Fig. 6a). To understand if FGF9 interfaces with BMP7 to regulate G1-S progression, we labelled NPCs treated with BMP7, FGF9, or BMP7 + FGF9 with CCNE1 and PCNA to distinguish cells in G1 and $S$ phases. BMP7 + FGF9 stimulation resulted in $\sim 50 \%$ fewer G1 and G1-S cells and 30\% more S-phase cells compared with BMP7 or FGF9 treatment suggesting that BMP7 and FGF9 promote NPC proliferation by accelerating the G1 to S cell cycle progression (Fig. 6b). To determine how FGF9 and BMP7 control the G1-S transition, we measured stimulation of the BMP7-
TAK1-JNK-JUN-controlled G1 regulatory genes Ccnd1, Ccnd3, $M y c$, Ccne1 and $C d c 25 a$ by either factor or both factors together. Expression of all five transcripts was upregulated by BMP7 and interestingly, FGF9 stimulation also increased their transcription indicating that FGF9 contributes to regulation of AP-1 targets. BMP7 + FGF9 combinatorial stimulation showed an additive effect on these targets, indicating that BMP7 and FGF9 coordinately control transcription of G1-phase cell cycle regulators (Fig. 6c and Supplementary Fig. 6c).

AP-1 function is regulated by dimer composition as well as the phosphorylation status of its constituents, and JUN-FOS 
a
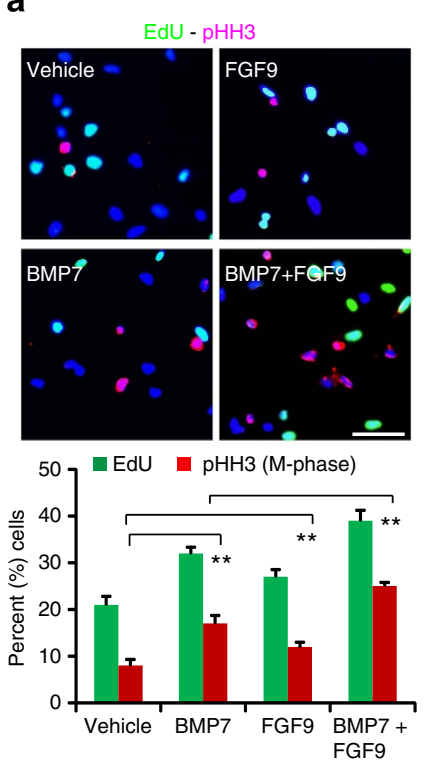

g

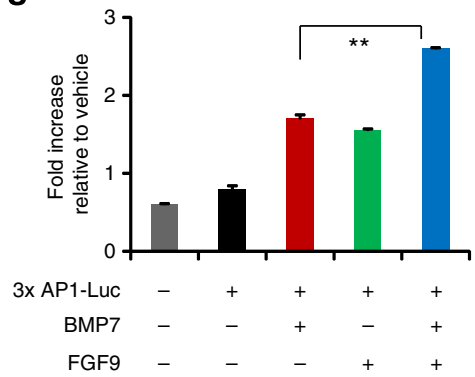

b

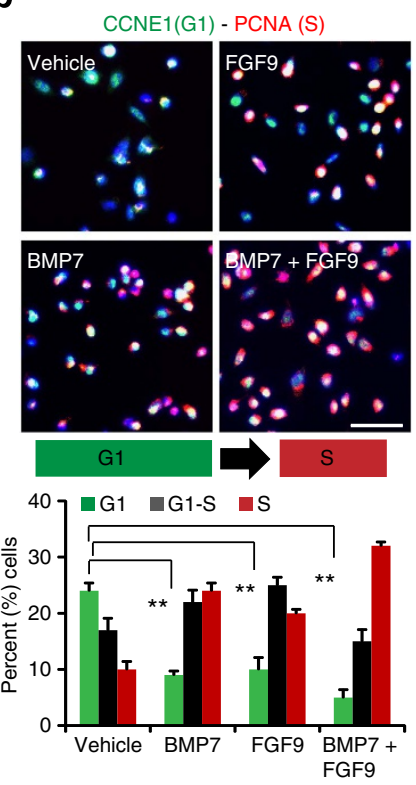

c

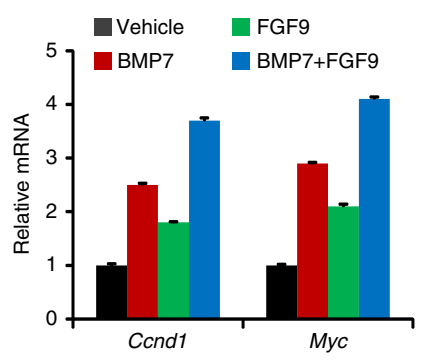

d

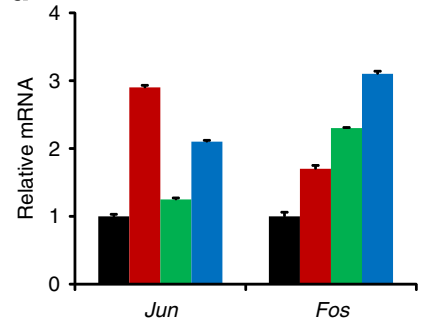

e

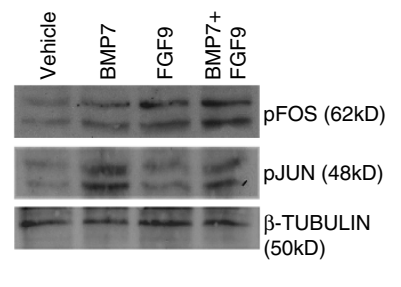

f

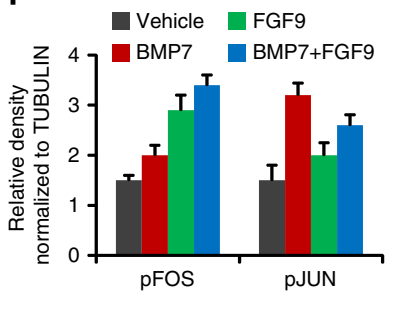

h

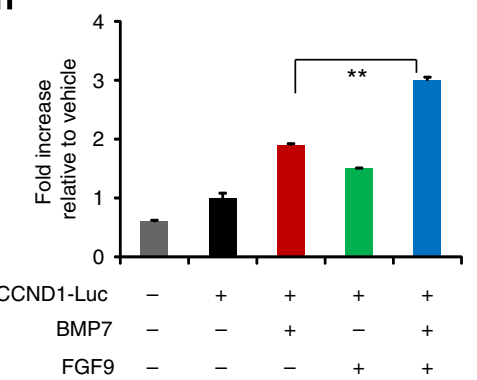

i

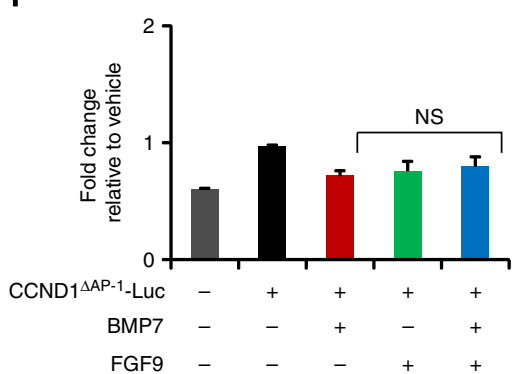

Figure 6 | Combinatorial BMP7 and FGF9 signalling controls AP-1 function in NPCs. (a) Immunostaining of EdU (S-phase) and pHH3 (M-phase), (b) CCNE1 (G1-phase) and PCNA (S-phase) in E17.5 NPCs stimulated with vehicle, BMP7, FGF9 or BMP7 + FGF9 for 24 h. Scale bars, $50 \mu M$. Graphs show the percentage of EdU +, $\mathrm{pHH} 3+$ cells and G1, G1-S and S-phase cells in each condition. Error bars represent mean (s.d.). ${ }^{\star \star} P<0.005$ and $P<0.001$ (Student's $t$-test). Two to three biological replicates analysed per condition ( $n=2$ in $\mathbf{a}$ ) and ( $n=3$ in $\mathbf{b})$ (c, d) RT-qPCR of cell cycle genes (Ccnd1 and Myc), Jun and Fos in NPCs stimulated with vehicle, BMP7, FGF9 or BMP7 + FGF9 for $2 \mathrm{~h}$. Error bars represent s.d. Three biological replicates analysed per condition, $n=3$. (e,f) pJUN and pFOS immunoblot of NPCs stimulated with vehicle, BMP7, FGF9 or BMP7 + FGF9 for 20 min. Graph shows the relative density of pJUN and pFOS normalized to $\beta$-tubulin in each condition (Supplementary Fig. 6e). (g-i) Bars in the graphs represent the average fold change in luciferase activity of 3XAP1-Luc, CCND1-Luc and CCND1 ${ }^{\triangle A P-1}$-Luc in NPCs stimulated with BMP7 and FGF9 relative to vehicle treatment for $24 \mathrm{~h}$. Three biological replicates analysed per condition $(n=3)$. Error bars represent s.d. ${ }^{\star \star} P<0.005$, NS, not significant $P>0.05$, Student's $t$-test.

heterodimers activate targets more efficiently than JUN homodimers 9 ,10. Given that BMP7 and FGF9 combinatorial stimulation increased transcription of G1-phase cell cycle regulators containing AP-1-binding elements, we speculated that FGF9 may modulate transcription and phosphorylation of JUN or its dimeric partner FOS concomitantly with BMP7 to regulate AP-1 function. We first measured the effects of FGF9 and BMP7 stimulation on Jun and Fos transcription. As expected, Jun transcription was upregulated by BMP7, but surprisingly it was unaffected by FGF9 (Fig. 6d). FGF9 stimulation of cells was verified by measuring expression of the FGF-target gene Spry1 (Supplementary Fig. 6d). Fos transcription, on the other hand, was strongly induced by FGF9 compared to BMP7 and this effect was further enhanced by combined stimulation with BMP7. Thus, while FGF9 and BMP7 cooperatively promote Fos transcription, the obligate DNA-binding partner Jun is controlled by BMP7 alone (Fig. 6d). Examination of JUN and FOS phosphorylation showed that BMP7 robustly activates JUN (3.15-fold), whereas FGF9 activates FOS (2.85-fold) (Fig. 6e and Supplementary Fig. 6e). BMP7 + FGF9 stimulation resulted in simultaneous phosphorylation of FOS and JUN suggesting that AP-1 transcriptional activation may be potentiated (Fig. 6f). To test this, we transfected NPCs with an AP-1 luciferase reporter $(3 \times \mathrm{AP} 1-\mathrm{Luc})$ and measured reporter activity in response to BMP7 and FGF9 stimulation ${ }^{27}$. BMP7 or FGF9 treatment resulted in less than a twofold luciferase response, but combined treatment caused more than a 2.5 -fold increase, indicating that simultaneous JUN and FOS activation promotes AP-1 transcriptional activity (Fig. 6g). To test this in a gene that directly influences proliferation in NPCs, we compared activation of a Ccnd1 luciferase reporter (CCND1-Luc) with a variant in which the AP-1-binding site has been mutated $\left(\mathrm{CCND} 1^{\triangle \mathrm{AP}-1}-\mathrm{Luc}\right)^{28}$. BMP7 or FGF9 treatment alone showed less than twofold luciferase response, whereas BMP7 + FGF9 stimulation resulted in a threefold increase, demonstrating that concurrent BMP7 and FGF9 signalling strongly promotes AP-1 function compared with either factor alone (Fig. 6h). Dependence on the AP-1 element for this transcriptional activity was confirmed by the finding that the Ccnd1 promoter with mutated AP-1-binding site was unresponsive to BMP7 and/or FGF9 stimulation (Fig. 6i).

We previously reported that transgenic expression of the FGF feedback regulator Spry1 in NPCs results in increased apoptosis 
in cap mesenchyme ${ }^{6,29}$. To confirm the contribution of FGF signalling to FOS regulation in vivo, we generated Six2-cre; Spry1-Tg mice. Kidneys were severely hypoplastic at P0, and body weight was reduced compared with WT littermate controls (Fig. 7a,b; and Supplementary Fig. 7a-c). Spry1-Tg kidneys revealed a thin nephrogenic zone with depleted cap mesenchymes and distended tubules (insets in Fig. $7 \mathrm{a}$ and Supplementary Fig. 7d). SIX2 immunostaining confirmed premature loss of NPCs in Spry1-Tg kidneys with $\sim 65 \%$ reduction in NPC number (Fig. 7d and Supplementary Fig. 7e). Spry1 expression increased sevenfold in Spry1-Tg NPCs, whereas the FGF-target gene Pea3 was reduced by $55 \%$, confirming inhibition of FGF signalling. Fos transcript diminished by $70 \%$, whereas $J u n$ and its upstream regulator Tak1 remained unchanged (Fig. 7e). To determine if Spry1-mediated attenuation of FGF signalling strictly results in reduced activation of FOS, we compared pFOS and pJUN expression in cap mesenchymes of P0 WT and Spry1-Tg versus $J_{u n}{ }^{\text {het }}$ and Jun ${ }^{N P C}$ kidneys. Expression of pFOS was reduced, whereas pJUN levels remained intact in cap mesenchymes of Spry1-Tg kidneys. Reciprocally, pFOS levels were unperturbed while pJUN was strongly reduced in cap mesenchymes of Jun NPC kidneys (Fig. $7 \mathrm{f}$ and Supplementary Fig. 7f). We next asked if reduced activation of JUN and FOS in $J u n^{N P C}$ and Spry1-Tg NPCs, respectively, decreases AP-1 transcriptional activity in response to BMP7 or FGF9 stimulation, and whether this effect can be rescued by expressing JUN or FOS in mutant NPCs. We treated $3 \times$ AP-1Luc-transfected E17.5 NPCs isolated from Jun het and $J u n^{N P C}$, WT and Spry1-Tg kidneys with BMP7 and/or FGF9. Interestingly, Jun-deficient NPCs failed to activate the AP-1 reporter in response to both BMP7 and FGF9 treatment, whereas Spry1-Tg NPCs only showed a slight reduction in AP-1 reporter activity in response to BMP7, but were completely unresponsive to FGF9 (Fig. 7g,h). Expression of a wild type JUN construct (pCMV-JUN) rescued AP-1 reporter activation in both BMP7and FGF9-stimulated Jun-deficient NPCs, and expression of a FOS phosphorylation-mimic construct (pcDNA-FOSDD) rescued AP-1 reporter activity in FGF9 stimulated Spry1-Tg NPCs (Fig. 7g,h) ${ }^{30,31}$. This suggests that JUN is essential for AP-1 activation by both growth factors, and the availability of FOS determines the amplitude of AP-1 activity. Cellular identity and transfection efficiency of NPCs was verified by examining cap mesenchyme and pre-tubular aggregate markers, expression of a GFP construct, JUN and pJUN immunostaining and RT-qPCR analysis of Jun transcript levels in transfected cells (Supplementary Fig. 7g-l). To determine whether JUN and FOS are required for the proliferative response of NPCs to BMP7 and FGF9, we performed EdU labelling of NPCs isolated from E17.5 $J u n^{\text {het }}$ and $J u n^{N P C}$, WT and Spry1-Tg kidneys and stimulated with BMP7 and/or FGF9. Jun-deficient NPCs failed to respond to both BMP7 and FGF9 stimulation. However, proliferation of Spry1-Tg NPCs in response to FGF9 was severely attenuated, and only slightly reduced in response to BMP7 suggesting that Jun is essential for proliferative response of NPCs to both BMP7 and FGF9 (Fig. 7i,j).

On the basis of these studies, we propose that BMP7 and FGF9 cooperatively control the composition of AP-1 dimers in NPCs, and that AP-1 composition influences the strength of activation of cell cycle regulators such as Ccnd1 (Fig. 7k).

\section{Discussion}

BMP7 is required for NPC maintenance in the developing kidney ${ }^{15-17}$. We show that the signalling cascade is initiated by activation of the MAPKKK TAK1 in response to BMP7 stimulation. TAK1 can be activated by numerous stimuli, but the finding that $B m p 7$ and Tak1 interact to regulate NPC renewal in vivo indicates an essential role for TAK1 specifically in the BMP7 pathway ${ }^{32}$. TAK1 activates JNK, which phosphorylates the transcription factor JUN, and the kinase activity of each of these components is essential for proliferation of NPCs. Kidneys lacking Tak1 or Jun in cap mesenchyme display identical phenotypes characterized by premature depletion of NPCs, indicating that JUN may be the sole essential mediator downstream of TAK1 in this signalling process. We show that $M y c$ is a transcriptional target of the BMP7-TAK1-JNK-JUN pathway in NPCs. MYC is essential for proliferation of cap mesenchyme in vivo and, together with JUN, activates G1-phase cell cycle regulators, explaining the proliferative effect of BMP7 stimulation ${ }^{14}$. NPCs display different average cell cycle lengths during early (E13.5) and later (E17.5) stages of nephrogenesis. Proliferation profiles of cap mesenchymes at these stages suggest that they are heterogeneous, containing both slowly and rapidly dividing cells ${ }^{33}$. An interpretation consistent with models of other stem/progenitor cell populations is that the slowly dividing subset may represent the self-renewing CITED1 + /SIX2 + population, whereas the rapidly dividing subset represents the CITED $1-/$ SIX2 + population that is differentiating ${ }^{34,35}$. Our finding that the CITED $1+/$ SIX $2+$ population is reduced following conditional inactivation of Tak1 using Six2-cre and Cited 1-creER $R^{T 2}$ drivers without any notable effect on proliferation of the CITED1-/SIX2 + population indicates that the BMP7-TAK1-JNK-JUN pathway is used primarily by the slowly dividing, self-renewing cells in the cap mesenchyme. The cycle length of NPCs increases as the embryo ages indicating that control mechanisms are added as development progresses. Interestingly, MYC targets are primarily regulated late in nephrogenesis, which is consistent with the observation that conditional $M y c$ inactivation slows cap mesenchyme proliferation only after E15.5. Whether cell cycle control through MYC late in nephrogenesis represents addition of a control mechanism or simply redundancy with N-MYC, whose expression is lost late in nephrogenesis will need to be answered by comparison of conditional inactivation of both factors ${ }^{36}$.

In addition to reduced proliferation in the cap mesenchyme, loss of Bmp7 causes ectopic cell death within the nephrogenic zone ${ }^{16-18}$. We do not see any effects on survival in kidneys in which Takl or Jun have been inactivated in the cap mesenchyme. However, inactivation of Smad4 using Bmp7-cre results in ectopic cell death within the nephrogenic zone of mutant kidneys, indicating that cell survival may be regulated through the SMAD pathway ${ }^{15}$. Mechanisms that control the balance between SMAD versus TAK1 signalling downstream of BMP7 in the cap mesenchyme are not currently understood. However, recent work indicates that FGF signalling may negatively regulate SMAD signalling providing an explanation for the lack of SMAD signalling seen in the CITED $1+$ compartment $^{37}$.

Composition of the AP-1 dimer is a critical factor in determining cellular fates such as proliferation, apoptosis and differentiation ${ }^{10}$. We find that BMP7 robustly controls transcription and activation of JUN, whereas FGF9 strongly induces transcription and phosphorylation of FOS. Analysis of the Six2-cre;Spry1-Tg mouse strain in which the FGF feedback inhibitor SPRY1 is expressed in NPCs and primary cell transcriptional reporter assays indicates that the activation of JUN and FOS by simultaneous BMP7 and FGF9 signalling potentiates AP-1 transcription, and we propose that co-regulation of the AP-1 transcription factor is one basis for the cooperative effect of BMP7 and FGF9 in kidney development. While we have defined the signalling cascade between BMP7 and Jun, the pathway between FGF9 and Fos is less clear. The inhibitory effect of Spry1 indicates that RAS is essential, and a recent report showing that NPC self-renewal is dependent on PI3K suggests 
that FGF9-RAS-PI3K may be the pathway governing Fos expression $^{38}$.

Although JUN can form homodimers to activate target transcription, these bind AP-1 elements less tightly than
JUN-FOS heterodimers, and have weaker transcriptional activity. In the case of BMP7 stimulation alone, the ratio would be skewed towards homodimer formation, whereas concurrent FGF9 signalling would skew the ratio towards JUN-FOS heterodimers, a

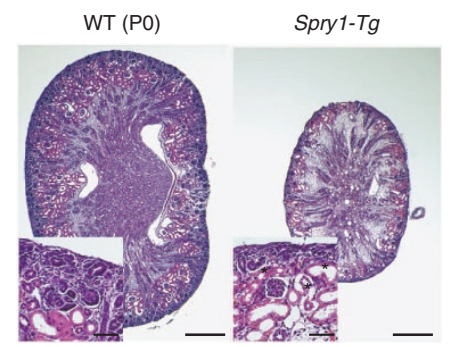

c
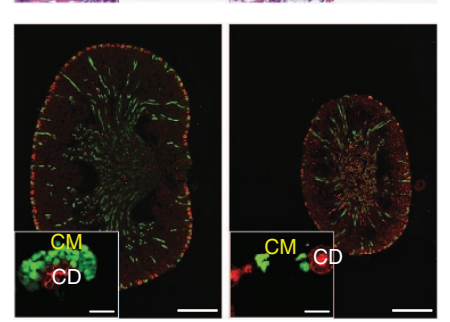

f

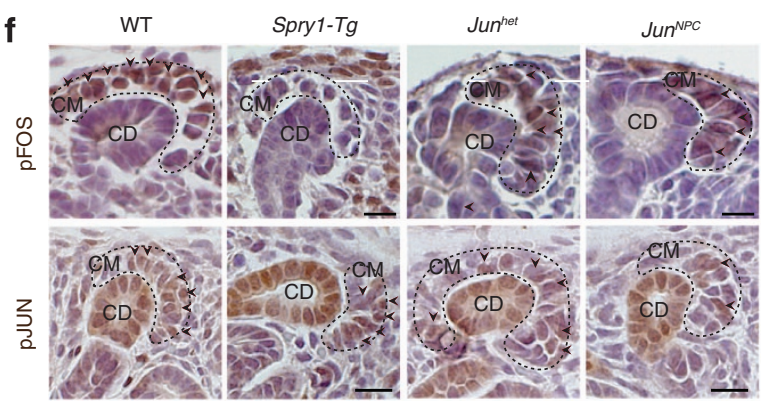

i
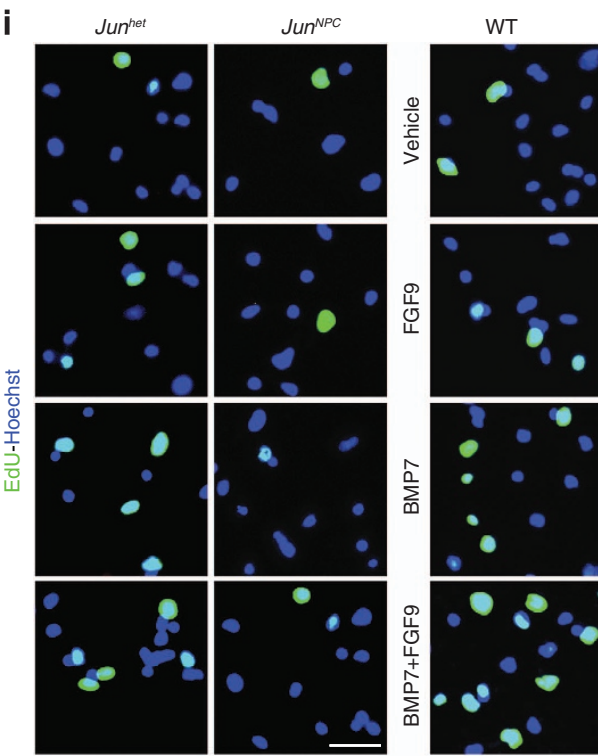

Spry $1-T g$
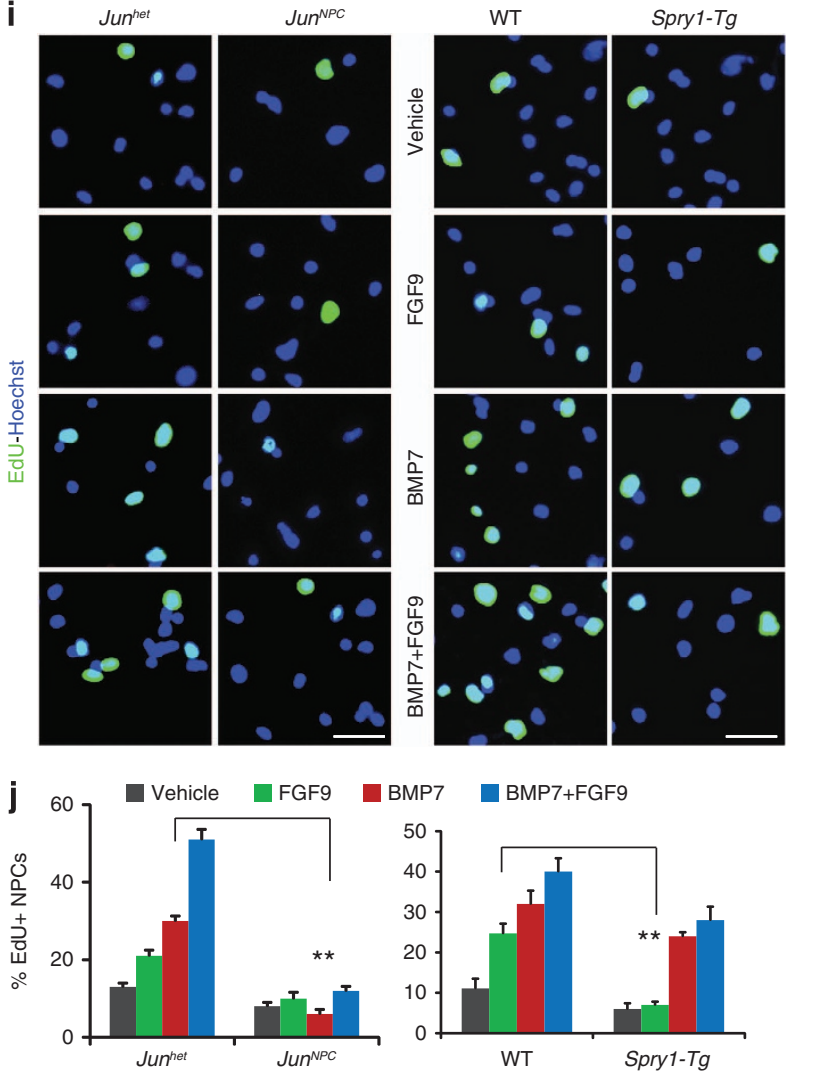
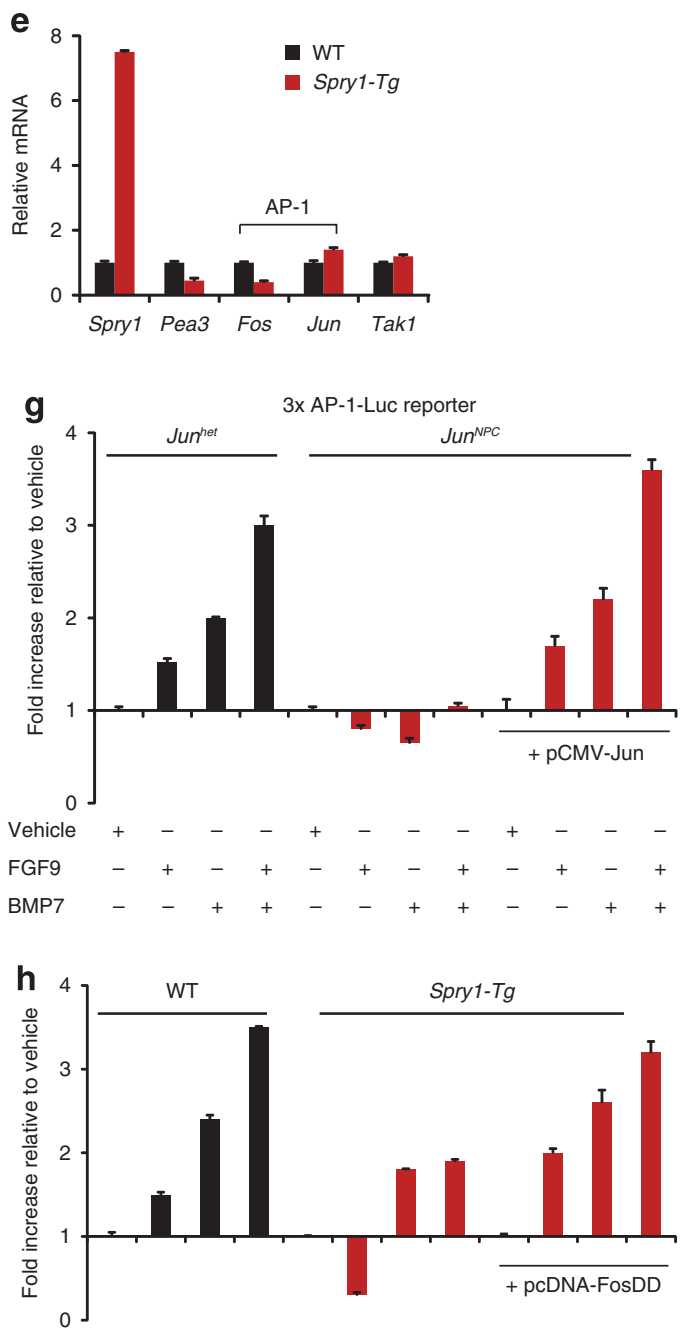

FGF9

BMP7

$\mathbf{k}$
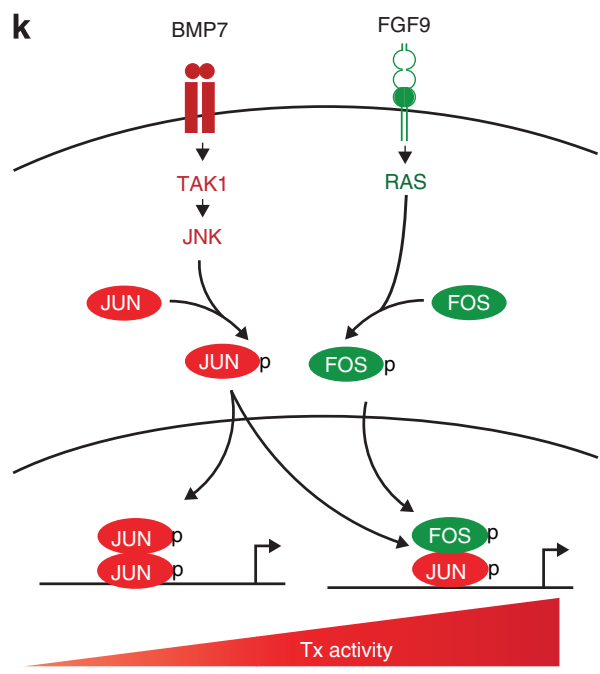
thus amplifying target transcription. Combinatorial BMP7 and FGF9 stimulation promotes robust transcription of the G1 regulator Ccnd1 in an AP-1-dependent manner, and we propose that control of AP-1 targets by combinatorial BMP7 and FGF9 signalling promotes G1-S transition, explaining a mechanism for the cooperative effects of these growth factors on NPC proliferation.

Our current findings identify AP-1 as a specific point of interaction of the BMP and FGF pathways in NPCs. We have previously shown that WNT and FGF activate common targets in $\mathrm{NPCs}^{6}$. It therefore seems possible that WNT9B- $\beta$-catenin signalling could converge with BMP7 and FGF9 on the regulation of AP-1. Signalling cross-talk between BMP, FGF and WNT pathways is a recurring theme in organogenesis, and $\mathrm{WNT} / \beta$-catenin signalling can regulate transcription of $\mathrm{AP}-1$ targets such as $M y c$, $C c n d 1$ and $C c n d 2$ (refs 39,40). Understanding this point of intersection further could explain the molecular basis for the combinatorial effects of these three distinct pathways on NPC proliferation.

\section{Methods \\ Mouse strains. Animal care was in accordance with the National Research Council Guide for the Care and use of laboratory animals and protocols were approved by the Institutional Animal Care and Use Committee of Maine Medical Center. Cited1-creER ${ }^{T 2}$ mice, R26RlacZ mice and Spry1-Tg mice were maintained on an FVB/NJ background ${ }^{29,41,42}$. Bmp $7^{+/ \text {cre }}$ mice were maintained on an ICR background. Six2-TGC $C^{t g}\left(\operatorname{Tg}(\text { Six2-EGFP/cre })^{1 A m c / J}\right), T_{a k 1}^{c / c}\left(\right.$ Map $\left.3 k 7^{t m 1.1 M d s}\right)$, $\mathrm{Tak} 1^{+/ c}$, and $J u n^{c / c}\left(J u n^{\text {tm }}{ }^{\text {wag }}\right)$ mice were maintained on the C57BL/6 background ${ }^{21,43,44}$. For tamoxifen-inducible cre mice, pregnant dams were injected at the indicated times with $3 \mathrm{mg}$ tamoxifen in corn oil per $40 \mathrm{~g}$ mouse.}

Plasmid constructs and adenoviral vector transduction. WT and kinasedefective TAK1 (K63W) plasmids were gifts from Dr Jun Ninomiya-Tsuji ${ }^{37}$ pCX-EGFP construct was a gift from Dr Andras Nagy ${ }^{45}$. FLAG-JUNWT-Myc and FLAG-JUN4A-MYC were gifts from Dr Axel Behrens (Addgene \# 47443, 47444) ${ }^{30}$. FOS-DD was a gift from Dr John Blenis (Addgene \# 8698) (11 $^{3} 3 \times$ AP1-pGL3 was a gift from Dr Alexander Dent (Addgene \# 40342) ${ }^{27}$. pGL3Basic-962 CCND1 promoter luciferase), pGL3Basic-962 CCND1 promoter AP-1 site mutant (Addgene \#32727 and \# 32728) were gifts from Dr Frank McCormick ${ }^{28}$. pRL-CMV (Renilla-Luciferase) was obtained from Promega. Adenoviral vectors (Ad-Cre and Ad-GFP) transduction was performed as described previously with a multiplicity of infection (MOI) of 500 for $40 \mathrm{~h}$ under serum-free conditions ${ }^{7}$.

Immunohistochemistry. Dissected kidneys were fixed in $4 \%$ paraformaldehyde (PFA) for $30 \mathrm{~min}$ (E14.5, E17.5 kidneys) to $1 \mathrm{~h}$ (P0 kidneys) at room temperature. Paraffin-embedded sections were incubated with blocking solution containing $1 \mathrm{X}$ phosphate buffered saline (PBS), 1\% bovine serum albumin, $5 \%$ serum of secondary antibody species (Jackson ImmunoResearch) and $0.05 \%$ hydrogen peroxide (Sigma) for $1 \mathrm{~h}$ at room temperature. Primary antibodies were diluted in 1X PBS and incubated at $4{ }^{\circ} \mathrm{C}$ overnight: anti-SIX2 (1:200-1:400, Proteintech), anti-CITED1 (1:200, Neomarkers), anti-GFP (1:200, Abcam), anti-LEF1 (1:100, Cell signaling), anti-pHH3 (1:100, Cell signaling), anti-Ki67 (1:200, Developmental Studies Hybridoma Bank (DSHB)), anti-caspase3 (1:100, Cell signaling), antiCytokeratin8/TROMA-I (1:200, DSHB), anti-DBA Lectin (1:500, Vector Laboratories), anti-JUN and anti-pJUN (1:200, Cell signaling), anti-FOS and anti-pFOS (1:400, Cell signaling), anti-CCND1 (1:100, Cell signaling), anti-CCND3 (1:100, Cell signaling), anti-MYC (1:100, Abcam), anti-CCNE1 (1:250, Santacruz) and anti-PCNA (1:200, Santacruz). Alexa-Fluor 488/568/647 conjugated secondary antibodies were used at 1:250 for detection of labelled cells. Nuclei were stained using DAPI (Molecular probes) for immunofluorescence and hematoxylin for immunohistochemistry. Sections were mounted using vectashield Mounting medium (Vector Laboratories). TUNEL staining was performed using ApopTagPlus peroxidase in situ apoptosis detection kit (EMD Millipore) according to manufacturer's instructions.

Purification of NPCs by magnetic bead depletion. Total NZCs were isolated from E14.5 and E17.5 ICR mice by enzymatic digestion as previously described ${ }^{7}$. For isolation of NZCs from E17.5 and P0 conditional mutants, control and mutant kidneys were sorted based on size and GFP expression (Six2-cre-EGFP and Cited1-creER $\left.{ }^{T 2}-E G F P\right)$, and confirmed by genotyping. Enrichment for CITED1 + cells and purification (referred to as NPCs) was performed by negative depletion with magnetic activated cell sorting using phycoerythrin-conjugated antibodies and anti-phycoerythrin Microbeads according to the manufacturer's protocol (Miltenyi Biotec $)^{2,11}$. Purified NPCs were cultured in monolayer in keratinocyte serum-free media (KSFM, Thermo Fisher Scientific) supplemented with rh-FGF2 (50 ng ml ${ }^{-1}$, $\mathrm{R} \& \mathrm{D}$ Systems) and $100 \mathrm{U} \mathrm{ml}^{-1}$ penicillin-streptomycin in plates coated with human plasma Fibronectin $\left(100 \mu \mathrm{g} \mathrm{ml}^{-1}\right.$, EMD Millipore). The identity of purified NPCs from ICR and conditional mutant mice was verified by immunostaining using anti-CITED1 (1:200, Cell Signaling), anti-SIX2 (1:200, Proteintech) and anti-LEF1(1:100, Cell Signaling) antibodies, and RT-qPCR analysis of cap mesenchyme and cortical interstitium markers before and after growth factor/inhibitor treatments.

Transfections and dual-luciferase reporter assays in NPCs. E17.5 NPCs cultured in KSFM (Thermo Fisher Scientific) media with rh-FGF2 $\left(50 \mathrm{ng} \mathrm{ml}^{-1}\right)$ were transfected for $24 \mathrm{~h}$ using lipofectamine 2000 (Life Technologies) ${ }^{24,25}$. Briefly, $1-2 \mu \mathrm{g}$ of plasmid DNA and 1-2 $\mu \mathrm{l}$ of lipid was mixed in a 1:1 ratio in Opti-MEM (Life Technologies) and added to NPCs in KSFM without antibiotics and incubated for $1 \mathrm{~h}$. Medium was replaced with fresh KSFM supplemented with rh-FGF2 $1 \mathrm{~h}$ after transfection to minimize cytotoxicity. Transfection efficiency was estimated using a pCX-EGFP construct at 24 and $48 \mathrm{~h}$ after transfection and by RT-qPCR for overexpressed genes. For luciferase reporter assays, transfected cells were stimulated with vehicle or indicated growth factors for $24 \mathrm{~h}$. Cells were lysed and luciferase activity was measured using the dual-luciferase reporter assay kit (Promega). Relative luciferase activity was normalized to Renilla-luciferase and average fold changes relative to vehicle treatment from four biological replicates and two independent experiments $(n=2)$ are represented in the graphs.

Immunoblotting. Isolated E17.5 NPCs were treated with rh-BMP7 or rh-FGF9 (50 and $100 \mathrm{ng} \mathrm{ml}^{-1}, \mathrm{R} \& \mathrm{D}$ systems), TAK1i $(0.5 \mu \mathrm{M}$, Analyticon Discovery) JNKi $(10 \mu \mathrm{M}$, Calbiochem) in KSFM (Thermo Fisher Scientific). Total protein was extracted as previously described ${ }^{7}$. Antibodies used were: anti-pTAK1 (Thr184/187), anti-pJNK (Thr183/Tyr185), anti-pJUN (S73), anti-pFOS (S32) (1:1,000, Cell signaling), anti- $\beta$-tubulin (1:5,000, Santa Cruz). Protein levels were quantified using FIJI/Image-J software by measuring the integrated density of the indicated proteins normalized to $\beta$-Tubulin, the loading control. Average values from two independent experiments $(n=2)$ are indicated in the graph.

Quantitative RT-PCR. RNA extraction from E14.5 and E17.5 NPCs was performed using RNeasy Microkit (Qiagen). Concentration of RNA was measured using NanoDrop2000 Spectrophotometer (Thermo Fisher Scientific), and a final concentration of 100-250 ng $\mathrm{l}^{-1}$ of RNA was used for cDNA synthesis by iScript Reverse Transcription Super Mix (BioRad). Quantitative RT-PCR was performed using iQ-SYBR Green Super mix (BioRad). Primer sequences of genes are listed in Supplementary Table 1. Fold changes were normalized to the housekeeping gene $\beta$-actin and average values (mean \pm s.d.) of three technical replicates and from two to three independent experiments $(n=2$ or 3$)$ are shown in the figures. $P$ values were calculated using a two-tailed Student's $t$-test, and $P<0.05$ was considered significant.

Figure 7 | Jun is essential for AP-1 activation by BMP7 and FGF9 in NPCs. (a) H\&E staining of PO WT (Six2-cre or Spry1-Tg littermate controls) and Spry1-Tg (Six2-cre;Spry1-Tg) kidneys. Insets show higher magnification images of the nephrogenic zone. Scale bars, 500 and $50 \mu M$. (b) Kidney size of PO WT and Spry1-Tg kidneys. ${ }^{\star \star \star} P<0.0005$, Student's $t$-test. Number of mice ( $n$ ) per genotype is noted in the graph. (c) SIX2 (red, cap mesenchyme) and DBA lectin (green, collecting duct) immunostaining of Spry7-Tg kidneys. Scale bars, 500 and $25 \mu$ M (d) Number of SIX2 + NPCs per section in PO WT and Spry1-Tg kidneys. Error bars represent s.d. ${ }^{\star \star \star} P<0.0005$, Student' $t$-test. (e) RT-qPCR of Spry1, Pea3 (FGF-target), Fos and Jun (AP-1 components) and Tak1 in E17.5 WT and Spry1-Tg NPCs. Two biological replicates analysed per genotype $(n=2)$. Error bars represent s.d. (f) Immunostaining of pFOS and pJUN (black arrows) on PO WT, Spry1-Tg and Jun het, Jun ${ }^{N P C}$ kidneys. Scale bars, $50 \mu \mathrm{M}$. (g,h) Luciferase activity relative to vehicle treatment in E17.5 Jun ${ }^{\text {het }}$, Jun NPC and WT, Spry1-Tg NPCs transfected with $3 \times$ AP1-Luc and pCMV-JUN or pcDNA-FOSDD and treated with BMP7, FGF9 and BMP7 + FGF9 for $24 \mathrm{~h}$. Error bars represent s.d. Three biological replicates analysed per condition $(n=3)$. (i,j) EdU labelling (green, proliferation marker) of E17.5 NPCs isolated from Jun het, Jun NPC and WT and Spry7-Tg kidneys and treated with vehicle, BMP7, FGF9 and BMP7 + FGF9 for 24h. Average number of EdU + NPCs scored in each condition. Two biological replicates analysed per condition $(n=2)$. Error bars represent s.d. ${ }^{\star \star} P<0.005$, Student's $t$-test. Scale bars, $50 \mu \mathrm{M}$. (k) Model for BMP7 and FGF9 collaborative regulation of NPC self-renewal. CD, collecting duct; CM, cap mesenchyme. 
Whole mount immunostaining. Dissected kidneys were fixed in $4 \%$ PFA for $10 \mathrm{~min}$ at room temperature and washed with $1 \mathrm{X}$ PBS for $5 \mathrm{~min}$ at $4{ }^{\circ} \mathrm{C}$. Kidneys were permeabilised with $1 \mathrm{X}$ PBS containing $0.1 \%$ Triton-X for $10 \mathrm{~min}$ at $4{ }^{\circ} \mathrm{C}$ followed by a wash in 1X PBS containing $0.01 \%$ Tween. Kidneys were incubated with blocking solution containing $1 \mathrm{X}$ PBS containing $0.01 \%$ Tween with serum of secondary antibody species for $8 \mathrm{~h}$. Primary antibodies to anti-SIX2 (1:200, Proteintech) and anti-cytokeratin8/TROMA-1 (1:100, DSHB) were diluted in blocking solution and added to the wells containing the kidneys and incubated for $24 \mathrm{~h}$ at $4^{\circ} \mathrm{C}$. Alexa-Fluor 488/568 secondary antibodies (Molecular Probes) were used at 1:250 and incubated for $24 \mathrm{~h}$ to detect staining in the kidneys.

Cell cycle marker analysis. NPCs were cultured in monolayer with rh-BMP7 and/or rh-FGF9 (50 and $100 \mathrm{ng} \mathrm{ml}^{-1}$, R\&D systems) in KSFM (Thermo Fisher Scientific) for $24 \mathrm{~h}$. Cells were fixed in $4 \%$ PFA and blocked in 1X PBS containing serum of the secondary antibody species, following which they were incubated in primary antibodies to CCNE1 and PCNA (1:200, Santacruz). Alexa-Fluor-488 (CCNE1) and Alexa-Fluor-568 (PCNA) secondary antibodies were used to visualize the staining. Images (5-8) were taken per well for each condition with a minimum of three biological replicates and three independent experiments $(n=3)$. Pooled images were analysed by Image-J and number of cells positive for G1 $(\mathrm{CCNE} 1+)$, G1-S ( CCNE1 + /PCNA +$)$ and S (PCNA +$)$ phases were counted and divided by the total number of DAPI + nuclei to determine the percentage of cells representing G1, G1-S or S-phase. Data are represented as percentage of G1 or S-phase cells in each condition.

EdU labelling of NPCs. E17.5 NPCs were cultured in monolayer with rh-BMP7 and rh-FGF9 (50 and $100 \mathrm{ng} \mathrm{ml}^{-1}, \mathrm{R} \& \mathrm{D}$ systems) in KSFM. Cultures were incubated with $20 \mu \mathrm{M}$ EdU (Click-iT EdU Alexa-Fluor 488 Imaging Kit, Life Technologies) $4 \mathrm{~h}$ after growth factor stimulation and pulse-chased for $20 \mathrm{~h}$. Fixation, permeabilization and Click-iT reaction was performed according to manufacturer's instructions. Cultures were incubated with anti-pHH3 (1:100, Cell Signaling) antibody for $1 \mathrm{~h}$ and Alexa-Fluor-568 secondary antibody was used to visualize the staining. Nuclei were stained with Hoechst 33342 (Life Technologies). Images (5-10)were taken per well for each condition with a minimum of three biological replicates from two independent experiments $(n=2)$. Pooled images were analysed by Image-J and number of EdU + (S-phase) and/or pHH3 + (Mitosis or M-phase) nuclei were counted and divided by the total number of nuclei to determine the percentage of cells in S- and M-phases. Data are represented as percentage of S- or M- phase cells in each condition.

Morphometrics and statistical analyses. (i) Kidney weight measurements were normalized to body weights to account for any differences in overall body size. Kidney size measurements were determined by calculating the cross-sectional area of the pole-to-pole distance on dissected whole kidneys using SPOT 5.1 imaging software. Measured kidney weights and sizes of individual animals per group are represented in scatter plots. (ii) Quantitation of NPC number and proliferation was performed manually for a minimum of five serial sections $100 \mu \mathrm{M}$ apart per kidney per genotype and the total number of individual or tamoxifen-treated mice $(n)$ analysed per time point is indicated in the figures. Error bars represent mean (s.d.) for each animal per experimental group. (iii) Growth curve analysis was conducted by counting cells using a hemocytometer for a minimum of three to five biological replicates per condition from three independent experiments. Two-tailed Student's $t$-test was performed for all statistical analyses and the resulting $P$ values are noted in the figures.

\section{References}

1. Mugford, J. M., Yu, J., Kobayashi, A. \& McMahon, A. P. High-resolution gene expression analysis of the developing mouse kidney defines novel cellular compartments within the nephron progenitor population. Dev. Biol. 333, 312-323 (2009).

2. Brown, A. C. et al. Role for compartmentalization in nephron progenitor differentiation. Proc. Natl Acad. Sci. USA 110, 4640-4645 (2013).

3. Carroll, T. J., Park, J. S., Hayashi, S., Majumdar, A. \& McMahon, A. P. Wnt9b plays a central role in the regulation of mesenchymal to epithelial transitions underlying organogenesis of the mammalian urogenital system. Dev. Cell 9, 283-292 (2005).

4. Karner, C. M. et al. Canonical Wnt9b signaling balances progenitor cell expansion and differentiation during kidney development. Development 138, 1247-1257 (2011).

5. Barak, H. et al. FGF9 and FGF20 maintain the stemness of nephron progenitors in mice and man. Dev. Cell 22, 1191-1207 (2012).

6. Brown, A. C. et al. FGF/EGF signaling regulates the renewal of early nephron progenitors during embryonic development. Development 138, 5099-5112 (2011).

7. Blank, U., Brown, A. C., Adams, D. C., Karolak, M. J. \& Oxburgh, L. BMP7 promotes proliferation of nephron progenitor cells via a JNK-dependent mechanism. Development 136, 3557-3566 (2009).
8. Dudley, A. T., Godin, R. E. \& Robertson, E. J. Interaction between FGF and BMP signaling pathways regulates development of metanephric mesenchyme. Genes Dev. 13, 1601-1613 (1999).

9. Karin, M., Liu, Z. \& Zandi, E. AP-1 function and regulation. Curr. Opin. Cell Biol. 9, 240-246 (1997).

10. Hess, J., Angel, P. \& Schorpp-Kistner, M. AP-1 subunits, quarrel and harmony among siblings. J. Cell Sci. 117, 5965-5973 (2004).

11. Brown, A. C., Muthukrishnan, S. \& Oxburgh, L. A synthetic niche for nephron progenitor cells. Dev. Cell 34, 1-13 (2015).

12. Angel, P., Hattori, K., Smeal, T. \& Karin, M. The Jun proto-oncogene is positively autoregulated by its product, Jun/AP-1. Cell 55, 875-885 (1988).

13. Iavarone, C. et al. The platelet-derived growth factor controls c-myc expression through a JNK- and AP-1-dependent signaling pathway. J. Biol. Chem. 278, 50024-50030 (2003).

14. Couillard, M. \& Trudel, M. c-Myc as a modulator of renal stem/progenitor cell population. Dev. Dyn. 238, 405-414 (2009).

15. Oxburgh, L., Chu, G. C., Michael, S. K. \& Robertson, E. J. TGF $\beta$ superfamily signals are required for morphogenesis of the kidney mesenchyme progenitor population. Development 131, 4593-4605 (2004).

16. Dudley, A. T., Lyons, K. M. \& Robertson, E. J. A requirement for bone morphogenetic protein-7 during development of the mammalian kidney and eye. Genes Dev. 9, 2795-2807 (1995).

17. Dudley, A. T. \& Robertson, E. J. Overlapping expression domains of bone morphogenetic protein family members potentially account for limited tissue defects in BMP7 deficient embryos. Dev. Dyn. 208, 349-362 (1997).

18. Luo, G. et al. BMP-7 is an inducer of nephrogenesis, and is also required for eye development and skeletal patterning. Genes Dev. 9, 2808-2820 (1995).

19. Goncalves, A. \& Zeller, R. Genetic analysis reveals an unexpected role of BMP7 in initiation of ureteric bud outgrowth in mouse embryos. PLOS ONE 6, 4 e19370 (2011).

20. Davies, J., Hohenstein, P., Chang, C. \& Berry, R. A self-avoidance mechanism in patterning of the urinary collecting duct tree. BMC Dev. Biol. 14, 35 (2014).

21. Kobayashi, A. et al. Six2 defines and regulates a multipotent self-renewing nephron progenitor population throughout mammalian kidney development. Cell Stem cell 3, 169-181 (2008).

22. Dang, C. V. c-Myc target genes involved in cell growth, apoptosis and metabolism. Mol. Cell. Biol. 19, 1-11 (1999).

23. Wisdom, R., Johnson, R. S. \& Moore, C. c-Jun regulates cell cycle progression and apoptosis by distinct mechanisms. EMBO J. 18, 188-197 (1999).

24. Schreiber, M. et al. Control of cell cycle progression by c-Jun is p53 dependent. Genes Dev. 13, 607-619 (1999).

25. Shaulian, E. \& Karin, M. AP-1 in cell proliferation and survival. Oncogene 20, 2390-2400 (2001).

26. Georgas, K. et al. Analysis of early nephron patterning reveals a role for distal $\mathrm{RV}$ proliferation in fusion to the ureteric tip via a cap mesenchyme-derived connecting segment. Dev. Biol. 332, 273-286 (2009).

27. Vasanwala, F. H., Kusam, S., Toney, L. M. \& Dent, A. L. Repression of AP-1 function: a mechanism for the regulation of Blimp-1 expression and $\mathrm{B}$ lymphocyte differentiation by the B cell lymphoma- 6 protooncogene. J. Immunol. 15, 1922-1929 (2002).

28. Tetsu, O. \& McCormick, F. Beta-catenin regulates expression of cyclin D1 in colon carcinoma cells. Nature 398, 422-426 (1999)

29. Yang, X. et al. Overexpression of Spry1 in chondrocytes causes attenuated FGFR ubiquitination and sustained ERK activation resulting in chondrodysplasia. Dev. Biol. 321, 64-76 (2008).

30. Aguilera, C. et al. c-Jun N-terminal phosphorylation antagonises recruitment of the Mbd3/NuRD repressor complex. Nature 13, 231-235 (2011).

31. Murphy, O. L., Smith, S., Chen, R., Fingar, C. D. \& Blenis, J. Molecular interpretation of ERK signal duration by immediate early gene products. Nat. Cell Biol. 2, 556-564 (2002).

32. Yamaguchi, K. et al. Identification of a member of MAPKKK family as a potential mediator of TGF- $\beta$ signal transduction. Science 270, 2008-2011 (1995).

33. Short, K. M. et al. Global quantification of tissue dynamics in the developing mouse kidney. Dev. Cell 29, 188-202 (2014).

34. Calegari, F., Haubensak, W., Haffner, C. \& Huttner, W. B. Selective lengthening of the cell cycle in the neurogenic subpopulation of neural progenitor cells during mouse brain development. J. Neurosci. 25, 6533-6538 (2005).

35. Mascré, G. et al. Distinct contribution of stem and progenitor cells to epidermal maintenance. Nature 489, 257-262 (2012).

36. Bates, C. M., Kharzai, S., Erwin, T., Rossant, J. \& Parada, L. F. Role of N-myc in the developing mouse kidney. Dev. Biol. 222, 317-325 (2000).

37. Motamedi, F. J. et al. WT1 controls antagonistic FGF and BMP-pSMAD pathways in early renal progenitors. Nat. Commun. 5, 4444-4458 (2014).

38. Lindstorm, N. O., Carragher, N. O. \& Hohenstein, P. The Pi3K pathway balances self-renewal and differentiation of nephron progenitor cells through $\beta$-catenin signaling. Stem Cell Rep. 4, 551-560 (2015). 
39. Guo, X. \& Wang, X. F. Signaling crosstalk between TGF- $\beta /$ BMP and other pathways. Cell Res. 19, 71-88 (2009).

40. Saadeddin, A., Babaei-Jabidi, R., Spencer-Dene, B. \& Nateri, A. S. The links between transcription, $\beta$-catenin/JNK signaling and carcinogenesis. Mol. Cancer Res. 7, 1189-1196 (2009).

41. Soriano, P. Generalized lacZ expression with the ROSA26 Cre reporter strain. Nat. Genet. 21, 70-71 (1999).

42. Boyle, S. et al. Fate mapping using Cited1-creERT2 mice demonstrates that the cap mesenchyme contains self-renewing progenitor cells and gives rise exclusively to nephronic epithelia. Dev. Biol. 313, 234-245 (2008).

43. Liu, H., Xie, M., Schneider, M. D. \& Chen, Z. J. Essential role of TAK1 in thymocyte development and activation. Proc. Natl Acad. Sci. USA 103, 11677-11682 (2006)

44. Behrens, A. et al. Impaired postnatal hepatocyte proliferation and liver regeneration in mice lacing c-jun in liver. EMBO J. 21, 1782-1790 (2002).

45. Okabe, M., Ikawa, M., Kominami, K., Nakanishi, T. \& Nishimune, Y. 'Green mice' as a source of ubiquitous green cells. FEBS Lett. 407, 313-319 (1997).

\section{Acknowledgements}

We thank Dr Jun Ninomiya-Tsuji for TAK1 and JUN constructs, Dr Michael Schneider and Dr Laurie Glimcher for Tak1 $1^{c / c}$ mice and Dr Axel Behrens for $J u n^{c / c}$ mice. This work was supported by National Institutes of Diabetes and Digestive and Kidney Disease Grant R01DK078161 (L.O.). Core facilities support was provided by Maine Medical Center Research Institute core facilities for molecular phenotyping and progenitor cell analysis (supported by National Institutes of General Medicine (NIGMS) 5P30 GM106391), Histopathology (NIGMS 5P30 GM106391 and 5P30 GM103392),
Transgenics and Gene Targeting (NIGMS 5P30 GM103392) and Cell Culture /Viral Vectors (NIGMS 5P30 GM103392).

\section{Author contributions}

S.D.M. and L.O. designed, conducted the experiments and analysed the data. X.Y. and R.F. provided reagents and mice. S.D.M. and L.O. wrote the manuscript. All authors discussed the results and commented on the manuscript.

\section{Additional information}

Supplementary Information accompanies this paper at http://www.nature.com/ naturecommunications

Competing financial interests: The authors declare no competing financial interests.

Reprints and permission information is available online at http://npg.nature.com/ reprintsandpermissions/

How to cite this article: Muthukrishnan, S. D. et al. Concurrent BMP7 and FGF9 signalling governs AP-1 function to promote self-renewal of nephron progenitor cells. Nat. Commun. 6:10027 doi: 10.1038/ncomms10027 (2015).

(c) (i) This work is licensed under a Creative Commons Attribution 4.0 International License. The images or other third party material in this article are included in the article's Creative Commons license, unless indicated otherwise in the credit line; if the material is not included under the Creative Commons license, users will need to obtain permission from the license holder to reproduce the material. To view a copy of this license, visit http://creativecommons.org/licenses/by/4.0/ 